\title{
Une lignée d'éditeurs-libraires montréalais, spécialisés en droit, au tournant du siècle
}

Sylvio Normand ${ }^{+}$

\section{Introduction}

L'histoire de l'édition juridique a donné lieu a bien peu de recherche. C'est probablement aux États-Unis que les études sont les plus avancées. ${ }^{\mathrm{I}} \mathrm{Au}$ Québec, l'histoire du commerce et de l'édition du livre de droit reste à faire. Il est possible de puiser ici et là un certain nombre de renseignements dans des études sur l'histoire de l'imprimé, ${ }^{2}$ mais jamais ces études ne mettent en exergue la production juridique, exception faite de l'édition des documents officiels qui a déjà donné lieu à des travaux importants. ${ }^{3}$ Par ailleurs, il faut préciser que des recherches sur l'histoire de la culture juridique québécoise fournissent tout de même des jalons utiles pour une mise en contexte de l'imprimé juridique. ${ }^{4}$

La présente recherche s'inscrit dans un projet consacré à l'étude de la culture juridique québécoise de 1840 à 1920 . Un volet de cette étude s'intéresse à la production éditoriale. Les données rassemblées sont encore trop limitées pour permettre de brosser un tableau complet du monde de l'édition juridique durant la période considérée. Toutefois, il est d'ores et déjà possible de jeter un certain éclairage sur une lignée de libraires-éditeurs montréalais qui se sont spécialisés dans le livre de droit.

Durant la seconde moitié du XIX ${ }^{\mathrm{e}}$ siècle, plusieurs éditeurs québécois lancèrent sur le marché des ouvrages et des périodiques de droit. Leur production éditoriale ne se limitait pas cependant au droit, mais embrassait un champ beaucoup plus vaste qui allait du livre scolaire, à la religion, en passant par la littérature.

À cette époque, les libraires-éditeurs spécialisés en droit étaient fort peu nombreux. Le marché était trop limité pour permettre à plusieurs maisons de survivre. Tout au plus était-il possible à une maison de s'implanter. Amédée Périard devint ainsi le premier libraire-éditeur à faire du commerce du livre de droit une spéciali-

† Sylvio Normand est professeur agrégé à la Faculté de droit de l'Université Laval. 
sation. À son décès, son commerce passa aux mains d'une société constituée par Camille Théoret et Patrick Whiteford. Cette société fut dissoute lorsque Théoret racheta la part de son associé. Par la suite, le commerce fut acquis par la société Wilson et Lafleur, qui se transforma plus tard en corporation. Cette dernière maison, qui existe toujours, assure donc la continuité d'une longue tradition. Ces libraires-éditeurs, même s'ils occupèrent une place non négligeable dans le monde du commerce du livre au tournant du siècle, sont à peine cités dans les ouvrages sur l'histoire de l'imprimé au Québec. $^{5}$

Cette étude vise à montrer comment s'est constituée, puis a évolué, une entreprise qui fit commerce de l'imprimé juridique. La documentation ayant servi à cette étude est parcellaire. Outre un inventaire de la production éditoriale (voir l'annexe), elle est surtout constituée de contrats, de correspondance et de documents corporatifs.

\section{Amédée Périard ou les bases d'une entreprise}

Le Montréalais Amédée Périard fut probablement le premier éditeur-libraire québécois à se spécialiser dans le commerce du livre de droit. Il se disait 'clerk' au début des années $1880 .^{6}$ Il était peut-être déjà en contact avec le monde du livre à titre de commis dans une librairie, mais rien ne permet de l'affirmer. En I 882, il se présentait comme 'law book publishers' agent ${ }^{\prime 7}$ et donnait sa résidence comme adresse. Il est vraisemblable qu'à cette époque, il agissait surtout comme intermédiaire de maisons d'édition étrangères.

C'est probablement en I 883 qu'il ouvrît, sur la rue Saint-Jacques, une librairie spécialisée en droit auquel il donna le nom de 'Librairie de droit et de jurisprudence,' nom qu'il changea plus tard pour celui de 'Librairie générale de droit et de jurisprudence,' nom identique à celui d'une grande maison parisienne de l'époque. Cette librairie était vraisemblablement la mieux fournie du genre à Montréal, au moins pour les ouvrages de langue française. Quatre catalogues, datant respectivement de $\mathrm{r} 883$ (Cat. $\mathrm{n}^{\circ} \mathrm{I} 34$ ), de $\mathrm{r} 885$ (Cat. $\mathrm{n}^{\circ} \mathrm{I} 35$ ), de 1890 (Cat. $n^{\circ}$ I 36) et de I 89I (Cat. $n^{\circ}$ I 37), permettent d'apprécier l'importance du fonds de librairie de Périard. À titre d'exemple, mentionnons que le catalogue de I 883 comptait 398 titres différents. 
D'après ces catalogues, la variété des titres offerts dans la librairie était impressionnante. En plus des ouvrages canadiens, Périard gardait en librairie un fonds considérable d'auteurs français, britanniques et américains. L'importation d'ouvrages de droit constituait un volet important des activités du libraire. Les ouvrages classiques des deux systèmes juridiques (droit civil et common law) y étaient disponibles; de nombreux traités de droit civil dont ceux d'Aubry et Rau, de Demolombe, de Laurent, de Marcadé et Pont, de Mourlon, d'Ortolan, de Toullier et finalement de Troplong figuraient aux côtés de Blackstone et de ses nombreuses éditions commentées. La production éditoriale américaine occupait aussi une place de choix, notamment en droit commercial et en droit criminel. Périard était au fait des récentes parutions étrangères, il mentionnait même des ouvrages à paraître dans ses catalogues.

Le libraire offrait également $\mathrm{d}^{\prime}$ anciennes publications françaises et canadiennes qu'il décrivait comme rares. Parmi celles-ci, citons un commentaire de la Coutume de Paris par Claude De Ferrière, Les loi civiles de Jean Domat, Le droit commun de la France de François Bourjon, le Traité sur les lois civiles du Bas-Canada de Henry Des Rivières Beaubien, et le Rapport des commissaires à la codification des lois civiles du Bas-Canada. L'intérêt pour des ouvrages portant sur l'Ancien droit français étant demeuré vivace même après la codification du droit privé en 1866 , il était normal que certains désirent enrichir leur bibliothèque $\mathrm{d}^{\prime}$ ouvrages épuisés. Le libraire pouvait certes se procurer ces ouvrages par l'intermédiaire de collègues européens. Il lui était également possible d'en récupérer en acquérant des bibliothèques lorsqu'un juriste québécois abandonnait la pratique ou mourait. ${ }^{8}$

L'éclectisme de cette librairie a de quoi surprendre le juriste contemporain. Des études antérieures ont déjà montré l'étendue de la culture juridique des praticiens de la fin du xIx ${ }^{\mathrm{e}}$ siècle. ${ }^{9}$ À cette époque, il était alors fréquent que les avocats dans leurs plaidoiries et les juges dans leurs jugements réfèrent à de nombreux ouvrages étrangers. Les bibliothèques privées de l'époque, dont nous possédons un certain nombre d'inventaires, ${ }^{10}$ reflétaient cette ouverture. Le contenu de la librairie de Périard, qui répondait certainement aux besoins de la clientèle, en est une preuve supplémentaire.

Les débuts des activités d'édition de Périard remontent à I 884 , si l'on fait exception d'un catalogue de librairie qu'il avait fait paraître l'année précédente. D'après notre inventaire, il lança quarante-etune publications durant sa carrière, soit une moyenne annuelle de presque cinq parutions (tableau I). Pour les fins de la présente étude, 
la production éditoriale a été répartie en six catégories (tableau 2). Les trois premières regroupent des publications à caractère surtout doctrinal, il s'agit des traités, des monographies et des manuels. Le traité, qui compte plusieurs tomes, présente une synthèse d'un domaine du droit. La monographie, moins ambitieuse, s'intéresse à un secteur plus limité d'une branche du droit ou porte sur une question précise. Le manuel, pour sa part, a pour but de faciliter l'étude du droit, il est surtout destiné aux étudiants en droit et en commerce. Les trois autres catégories suivantes rassemblent essentiellement des ouvrages de consultation. Les ouvrages de référence comprennent des index et des formulaires, dont la complexité peut varier considérablement. Les recueils d'arrêts rassemblent des jugements publiés in extenso. ${ }^{\text {II }}$ Finalement, les codes et lois présentent un texte législatif souvent accompagné de commentaires ou d'annotations.

Périard privilégiait la production d'ouvrages de consultation aux ouvrages doctrinaux. Ce type d'ouvrages, en plus de trouver preneur facilement, permettait souvent de multiples tirages. Les éditions de textes législatifs (Code civil, Code de procédure civile, Code municipal, et certaines autres lois), furent donc nombreuses: dix-huit au total. Le marché des éditions des codes, notamment ceux de petits formats, était probablement le plus assuré. Bon an mal an, un éditeur pouvait compter en écouler plusieurs exemplaires auprès des juges, des praticiens et des étudiants en droit. La plupart des maisons d'éditions s'efforçaient d'occuper le champ en produisant chacune ses propres codes. Ainsi, les éditeurs Charles-Odilon Beauchemin, ${ }^{12}$ Joseph-Moïse Valois, ${ }^{13}$ et la compagnie Carswell de Toronto $^{\mathrm{I} 4}$ mirent en vente des codes en même temps que ceux de Périard.

Outre les codes et les textes législatifs, les ouvrages de consultation comptaient aussi quelques recueils d'arrêts (Cat. $\mathrm{n}^{\circ} 29-3 \mathrm{I}$ ) et ouvrages de référence (Cat. $\mathrm{n}^{\circ} 2,5,8,9$ et 23 ) dont les recueils de formules notariales de Charles Cushing et de Félix-Gabriel Marchand, de même que le fameux répertoire de Jean-Joseph Beauchamp, qui présentait des résumés des arrêts du Conseil privé. Ces deux derniers ouvrages, qui avaient été certainement coûteux à produire, parurent en I $89 \mathrm{I}$. Ils furent des succès de librairie, puisqu'on les réimprima peu de temps après leur parution.

Malgré tous les aléas que pouvait réserver la publication d'une monographie, étant donné que le public des juristes était restreint et peut-être encore méfiant à l'endroit d'une doctrine québécoise, Périard édita tout de même pas moins de quatorze monographies. 
La majorité de ces publications présentait l'état du droit sur un point donné. Les secteurs du droit couvert par les auteurs de monographies sont étendus: droit civil $\left(\right.$ Cat. $\mathrm{n}^{0} 7,20,28$ et 36$)$, droit judiciaire (Cat. $\mathrm{n}^{\circ} 2$ I et 32), droit commercial (Cat. $\mathrm{n}^{\circ}$ ro), droit criminel (Cat. $\mathrm{n}^{\circ} 6$ ), et droit constitutionnel (Cat. $\mathrm{n}^{\circ}$ I 5 et 27 ). Quelques ouvrages tranchent par rapport au reste de la production. L'édition du Traité des substitutions de Claude-François Thévenot $\mathrm{d}^{\prime}$ Essaule de Savigny par Michel Mathieu (Cat. $\mathrm{n}^{\circ}{ }_{36}$ ) fait figure d'incongruité. L'ouvrage traitait d'une institution de droit civil disparue en France, mais encore présente dans le droit québécois et sur laquelle la documentation se faisait rare à la fin du $\mathrm{xIx}^{\mathrm{e}}$ siècle. L'adaptation de ce traité cherchait donc à combler un besoin auquel la doctrine française contemporaine ne répondait pas. En marge de cette production à caractère nettement professionel, Périard édita aussi des ouvrages sur l'histoire du droit (Cat. $\mathrm{n}^{\circ}$ I, 3, I3 et I4), dont l'Histoire $d u$ droit canadien depuis les origines de la colonie jusqu'à nos jours $\mathrm{d}^{\prime}$ Edmond Lareau, qui est resté un classique.

Périard publia les oeuvres de vingt-quatre auteurs différents. Il s'attacha même quelques uns d'entre eux qui firent paraître plus d'une oeuvre chez lui; Michel Mathieu, Thomas K. Ramsay, et Robert $S$. Weir en publièrent chacun deux, William P. Sharp et William A. Weir trois, Edmond Lareau quatre, et finalement Léon Lorrain six. Ces auteurs englobaient à eux seuls plus de la moitié de la production.

La plus grande part de la production éditoriale de Périard était destinée aux juristes québécois. Ainsi, tout ce qui avait trait au droit civil ne pouvait avoir grand intérêt hors de la province. Quelques ouvrages, rédigés en anglais, cherchaient cependant à rejoindre une clientèle plus vaste, il s'agit de la monographie de Charles M. Holt sur le droit canadien des chemins de fer (Cat. $\mathrm{n}^{0}$ Io) et des ouvrages de consultation de Jean-Joseph Beauchamp (Cat. $\mathrm{n}^{\circ}$ 2), de JosephFéréol Dubreuil (Cat. $\mathrm{n}^{\circ} 8$ ), de Joseph Frémont (Cat. $\mathrm{n}^{\circ}$ 9), et de Robert S. Weir (Cat. $\mathrm{n}^{0} 38$ ). Le recueil de Beauchamp connut probablement une certaine diffusion en Grande-Bretagne et dans les colonies britanniques. ${ }^{\text {I5 }}$

À l'instar des autres éditeurs, Périard trouvait probablement avantage à prendre en charge un périodique juridique. Une telle publication, en plus de lui accorder une visibilité constante auprès de sa clientèle, lui assurait une entrée régulière de fonds grâce aux abonnements. À partir de I884, il agit donc comme éditeur et diffuseur de la Revue légale pour le compte du juge Michel Mathieu, puis, en $\mathrm{I} 887$, il se portait acquéreur du périodique laissant à 
Mathieu la tâche de rédacteur en chef. ${ }^{16}$ En I 892, il décida de lancer une nouvelle revue le Monthly Law Digest and Reporter et en confia la rédaction à $\mathrm{F}$. Longueville Snow, alors bibliothécaire de la New York Life Law Library à Montréal. ${ }^{17}$ Les deux périodiques adoptaient une forme differente et ne visaient visiblement pas le même public. Le premier, qui s'adressait aux civilistes québécois, publiait intégralement des arrêts rendus par des tribunaux québécois. Le second cherchait à rejoindre une plus vaste audience, soit les avocats canadiens spécialisés en droit commercial. Le rédacteur sélectionnait des affaires en provenance du Canada et de l'étranger et en publiait un résumé.

Périard réimprima des tomes épuisés de périodiques juridiques. Car, il faut rappeler qu'en droit, les revues, surtout celles qui publient les jugements des tribunaux, conservent longtemps de l'intérêt auprès des praticiens et des juges, qui y trouvent des précédents qu'ils invoquent au soutien de leurs arguments dans leurs plaidoiries et leurs décisions. La demande pour d'anciens volumes, sinon pour des séries complètes de périodiques, pouvait donc être soutenue même après dix ou vingt ans. De nouvelles copies des cinq premiers volumes de la Revue légale, qui dataient de 1869 à $18733^{18}$ et la dernière parution du Lower Canada Reports, qui remontait à $\mathrm{I} 867,{ }^{19}$ furent donc tirées à nouveau. Un tel projet était certainement coûteux, puisque les planches originales étaient disparues et qu'il fallût recomposer en entier chacun des tomes. De plus, ces volumes étant, en droit, de véritables ouvrages de référence, Périard devait veiller à ce que la mise en page d'origine soit à peu près respectée de manière à rendre possible le renvoi à des passages précis; ce qui fut fait.

À l'instar des autres libraires montréalais, Périard faisait aussi commerce de papeterie et vendait les diverses formules utilisées par les praticiens. ${ }^{20}$ Dans sa publicité, il se présentait non seulement comme libraire et éditeur, mais aussi comme relieur. Les outils, inventoriés lors de son décès, confirment que lui-même ou un employé s'adonnait à cette activité à sa place d'affaire. En bon libraire, il avait alors avantage à offrir un tel service, puisque plusieurs de ses clients devaient être abonnés à des périodiques qui leur parvenaient en fascicules à raison d'une dizaine de parutions par année. La reliure permettait d'assurer une meilleure conservation de ces revues appelées à être consultées fréquemment. Périard devait aussi relier les ouvrages qu'il éditait. Il semble, en effet, $\mathrm{d}$ 'après deux contrats d'impression liant Périard et Charles-Odilon Beauchemin, que la tâche de l'imprimeur se limitait à transmettre 
l'ouvrage imprimé en feuilles à l'éditeur qui devait prendre en charge la reliure. Il est certain que Périard procédait à la reliure au fur et à mesure des ventes, puisque, d'après son inventaire après décès, il possédait plusieurs exemplaires en feuilles des ouvrages qu'il avait édités. ${ }^{21}$ L'intérêt montré par les juristes pour des ouvrages reliés n'avait d'ailleurs pas uniquement pour but d'assurer une bonne conservation des volumes, les praticiens y tenaient aussi parce qu'ils tiraient du prestige à posséder une importante bibliothèque propre à impressionner leurs clients.

Périard occupa brièvement le $7 \mathrm{I}$ de la rue Saint-Jacques ${ }^{22}$ pour s'installer, dès $1884,{ }^{23}$ au numéro 23 de la même rue. Quelques années plus tard, ses affaires ayant prospéré, il occupa également les numéros 2 I et $25 .{ }^{24}$ Périard ne devint cependant jamais propriétaire de son lieu de commerce, pas plus que de sa maison privée. La librairie logeait à un emplacement avantageux puisqu'elle était au coeur de la communauté juridique, le Palais de Justice étant à l'époque situé à quelques pas de là, de même que plusieurs cabinets d'avocats.

Il est difficile de déterminer l'importance du personnel de l'entreprise. Outre un relieur, dont les services n'étaient peut-être retenus qu'à temps partiel, la librairie devait compter sur un ou deux commis. Des actes notariés, par lesquels Périard accordait à des personnes désignées comme commis des procurations les autorisant à poser des actes courants de gestion, permettent d'identifier certains d'entre eux, soit: Alfred Turgeon, ${ }^{25}$ Camille Théoret, ${ }^{26}$ et Théophile Lafleur. ${ }^{27}$ Les deux derniers occupèrent par la suite une place importante dans le monde de la librairie et de l'édition juridiques montréalaises. Par ailleurs, deux professionnels semblent avoir joui de la confiance de Périard: le notaire Léandre Bélanger et l'avocat Louis-Wilfrid Sicotte. Ce dernier s'était d'ailleurs vu accorder une procuration munie de pouvoirs très étendus sur les affaires de l'entreprise. ${ }^{28}$

Nul doute que la clientèle de la librairie se recrutait pour un grande part dans le monde de la pratique du droit. La liste des débiteurs au décès de Périard est éclairante à cet égard, elle comptait pas moins de 256 personnes qui lui étaient redevables d'une somme totale de 2 I 98,85 \$. Les créances, relativement peu élevées, en moyenne $8,59 \$$, comprenaient sans nul doute plusieurs abonnements. La très grande part des débiteurs étaient domiciliés à Montréal (126) et dans la ville de Québec (vingt-et-un). Il n'en demeure pas moins que le libraire avait des débiteurs dans quarante-neuf autres villes québécoises, dont plusieurs étaient les chefs-lieux des districts 
judiciaires. Hors du Québec, Périard avait aussi quelques clients, en Ontario (à Ottawa et Toronto) aux États-Unis (à Albany, Boston, Jersey City, New York, Philadelphia, Rochester, et Worchester) et en Angleterre (Londres). Au nombre des débiteurs se trouvaient plusieurs praticiens du droit, des juges et quelques cabinets.

Un libraire-éditeur devait pouvoir compter sur plusieurs artisans et fournisseurs. La liste des créanciers, fournie par l'inventaire après décès de Périard, montre qu'il faisait affaire avec les imprimeurs montréalais suivants: Gebhart \& Berthiaume, Eusèbe Senécal, Joseph-Moïse Valois, la Gazette Printing Company, John Lovell \& Son, et Perreault Printing. Ses nombreuses importations de livres de droit le mettaient en contact avec plusieurs collègues de l'étranger dont E. Bruylant de Bruxelles, A. Giard et H. Bailieu de Paris, Baker, Voorhis \& Company de New York, ${ }^{29}$ Boston Book Company de Boston, Callaghan Company de Chicago, et Carswell de Toronto. Périard ne se portait pas nécessairement acquéreur des ouvrages étrangers qu'il présentait en librairie, il recevait plutôt en dépôt ${ }^{3 \circ}$ les nouveautés et devait renvoyer par la suite les invendus.

Périard s'était marié en septembre $\mathrm{I} 882$ avec Annie Maher à l'église St Mary's Church of England d'Hochelaga. ${ }^{31}$ Il décéda prématurément, en octobre I 892 , alors qu'il n'était âgé que de 39 ans. ${ }^{32}$ Son épouse et trois jeunes enfants lui survécurent. La cause de sa mort reste inconnue. Il existe peut être un lien entre son décès et un accident dont il avait été victime en mars de la même année, alors que la chute de glace d'une toiture l'avait affecté sérieusement. ${ }^{33}$

L'inventaire après décès de Amédée Périard évaluait à 30 523,39 \$ la valeur de son fonds de librairie. Cette évaluation, même si elle a été établie sous la supervision d'experts, était nettement excessive. En effet, la valeur des invendus fut établie en tenant compte uniquement de leur prix de vente et non de leur valeur marchande. De sorte que, par exemple, le stock, fort important, d'anciens numéros de périodiques que Périard conservait contribua sensiblement à augmenter la valeur du fonds, alors qu'il était impossible de trouver preneur, même à long terme, pour les dizaines ou même parfois les centaines d'exemplaires de certains numéros qu'il possédait. Les dettes de Périard s'élevaient à I2 722,9I \$, répartis entre 8I créanciers. En revanche, ses débiteurs lui étaient redevables de la somme de 2 I $98,85 \$$.

À première vue, la situation financière de la succession de Périard paraissait enviable. Elle était pourtant loin de l'être. L'actif était moins attrayant qu'il ne semblait et le passif avait été nettement sous-estimé. La succession fut donc acculée à la faillite. Les 
créanciers, après qu'ils eurent été invités à donner leur avis sur la nomination d'un curateur, ${ }^{34}$ produisirent des réclamations pour un montant total de $22482 \$ 35$ Deux banques figuraient en bonne place dans la liste des créanciers: la Banque Ville-Marie pour 5397 \$ et la Banque Nationale pour $2409 \$$. Contrairement à ce que l'on pourrait croire, le financement d'une entreprise d'édition auprès du secteur bancaire était donc relativement aisé. Des imprimeurs possédaient aussi des créances fort élevées; Gebhart \& Berthiaume réclamait 3582 \$, Joseph-Moïse Valois 729 \$, Eusèbe Senécal 722 , la Gazette Printing Company 405 \$, John Lovell \& Son 324 , et Perreault Printing $264 \$$. Des montants moins importants étaient aussi dus à certains auteurs et rédacteurs de revues; Jean-Joseph Beauchamp évaluait sa créance à $\mathrm{I} 000 \$$, William P. Sharp à $175 \$$, F. Longueville Snow à I $25 \$$, et Charles H. Stephens, l'éditeur d'un des ouvrages de Thomas K. Ramsay, à $585 \$$. Les biens de Périard furent vendus lors d'un encan public tenu à Montréal. ${ }^{36}$

Faute d'éléments de preuve, il est hasardeux de tenter d'expliquer les difficultés financières de Périard. De multiples facteurs peuvent avoir joué à son détriment. À l'époque où il lança son entreprise, il dut affronter de solides concurrents. Des imprimeurs-éditeurs polyvalents, tels que Charles-Odilon Beauchemin, Dawson Brothers, Eusèbe Senécal, et John Lovell, occupaient alors une place non négligeable dans l'édition juridique. Malgré son dynamisme, Périard $\mathrm{n}^{\prime}$ eut pas le temps, en dix ans, d'occuper le champ sans partage. L'examen de son inventaire après décès semble révéler qu'il supportait des inventaires importants de certains des livres qu'il avait édités. L'échec de quelques titres a pu compromettre la santé financière de son entreprise. En outre, même s'il a, semble-t-il, obtenu aisément du crédit, les trois dernières années d'activité de Périard dans l'édition engouffrèrent certainement des sommes considérables, puisqu'il publia dans cette brève période la moitié de sa production totale.

\section{L'intérim de Whiteford et Théoret}

Le fonds de commerce, composé de l'achalandage, de l'ameublement de bureau et du magasin et, bien sûr, des livres en librairie de Périard, fut adjugé pour la somme de 5234,83 \$a Camille Théoret. Ce fonds avait été évalué à I $4200 \$$ par le curateur de la faillite. Théoret, qui se présentait comme commis-libraire, prétendait agir pour le compte de Bernard Ewan McGale, marchand-drugiste de 
Montréal. Ce dernier, qui passa l'acte de vente avec le curateur aux biens de la succession le 5 avril $1893,{ }^{37}$ n'était là que pour assurer le financement de l'opération, puisqu'il revendit aussitôt le tout à Camille Théoret et à Patrick Whiteford, qui furent désignés à l'acte comme des marchands-libraires. ${ }^{38}$

Whiteford et Théoret, qui occupèrent la place d'affaire laissée vacante par Périard, signèrent un contrat de société le jour même où ils firent l'acquisition du fonds de commerce. ${ }^{39}$ Des deux associés, Théoret était probablement le plus expérimenté dans ce secteur d'activité ou le plus compétent en gestion, puisque le contrat de société prévoyait que c'était lui qui était chargé de tenir la comptabilité de l'entreprise jusqu'à parfait paiement des sommes dues à McGale.

L'établissement d'Amédée Périard jouissait certainement d'une bonne réputation à son décès, puisque Whiteford et Théoret $\mathrm{n}^{\prime}$ hésitèrent pas à inscrire sur leur papier à lettre qu'ils étaient ses successeurs en affaires. Les deux associés poursuivirent sur la lancée de leur prédécesseur, faisant rapidement preuve de dynamisme. Quelques mois après avoir acquis l'entreprise, ils distribuèrent un catalogue des ouvrages disponibles à leur librairie (Cat. $\mathrm{n}^{0}{ }_{13} 38$ ).

Pendant leur association, ils éditèrent sept volumes (Cat. $\mathrm{n}^{\circ} \mathbf{4 2}^{2}$ 48). Le Code criminel étant entré en vigueur en I892, le droit criminel devint un sujet d'intérêt (Cat. $\left.\mathrm{n}^{\circ} 44-45\right)$. C'est cependant une publication de droit civil qui permit à l'entreprise d'affirmer son leadership dans le monde de l'édition juridique. En effet, elle lança en 1895 , le premier tome du traité de droit civil de Pierre-Basile Mignault, le Droit civil canadien, qui rapidement allait devenir un classique (Cat. $\mathrm{n}^{\circ}$ 48). La publication des neuf tomes s'échelonna sur une vingtaine d'années. En marge de leurs travaux d'édition, ils réimprimèrent l'ouvrage de Jean-Joseph Beauchamp (Cat. $\mathrm{n}^{0}{ }^{2}$ ) sur la jurisprudence du Conseil privé qu'avait édité Périard en I891 et qui avait été épuisé après deux ou trois ans..$^{\circ}$

Intéressés à ajouter une autre revue de droit à leur production, Whiteford et Théoret acquirent de Charles-C. de Lorimier, médecinvétérinaire et fils du juge Charles-Chamilly de Lorimier, la Revue de jurisprudence qui venait tout juste d'être lancée. ${ }^{4 \mathrm{I}}$ Le juge, figure dominante de la scène judiciaire montréalaise, qui avait agi en tant que rédacteur pour cette revue, s'engageait à assumer les mêmes fonctions par la suite. En revanche, ils ne continuèrent pas l'édition du Monthly Law Digest and Reporter qu'avait lancé Périard en I 892 et qui ne dura qu'un an. 


\section{Camille Théoret, un homme d'affaire prospère}

La société Whiteford et Théoret fut de courte durée. Après un peu plus de deux ans seulement, les associés procédèrent à sa dissolution et Théoret acquit les droits de Whiteford. ${ }^{22}$ Il quitta probablement presque aussitôt les locaux qu'il occupait jusque là pour aménager, juste à côté, aux numéros I I et I 3 de la rue Saint-Jacques.

La production éditoriale de Camille Théoret s'apparente à celle de Périard. Durant les quelque onze ans où il fut actif, il édita cinquante-trois ouvrages (tableau I). La répartition entre les différents secteurs est même assez semblable dans les deux cas (tableau 2). Les ouvrages de consultation demeurent un peu plus nombreux que les ouvrages de doctrine. Les codes et les lois, au nombre de vingt-quatre, conservent une place de choix dans la production de Théoret. Certains auteurs en firent d'ailleurs leur marque de commerce. Joseph-Édouard Bédard, Oscar-Pierre et Albert-Paul Dorais, Paul-G. Martineau et Romuald Delfausse, William Prescott Sharp, et Robert Stanley Weir virent, en effet, à éditer et maintenir à jour les textes législatifs les plus utilisés par les juristes. Les ouvrages de référence se limitent à un index $\left(\right.$ Cat. $\left.\mathrm{n}^{\circ}{ }_{58}\right)$ et deux formulaires (Cat. $\mathrm{n}^{\circ} 7 \mathrm{I}$ et 72 ). La doctrine maintenait malgré tout une bonne place. Le droit commercial (Cat. ${ }^{\circ} 49,62,73$ et 99 ) et le droit judiciaire (Cat. $\mathrm{n}^{\circ} 59,77,78$ et 9 I) sont les deux domaines privilégiés, suivi du droit civil (Cat. $\mathrm{n}^{\circ} 74$ et 93 ), du droit criminel (Cat. $\mathrm{n}^{\circ} 63$ ), du droit international (Cat. $\mathrm{n}^{0} 75$ ) et du droit social (Cat. $\mathrm{n}^{0}$ 94). L'histoire du droit demeure aussi un champ d'intérêt (Cat. $\mathrm{n}^{\circ} 79$ et 87 ). Le manuel occupa une place importante dans sa production éditoriale. Les formules adoptées pour ce type d'ouvrages varient. La recherche de méthodes facilitant l'étude de la matière par les étudiants est évidente, comme en font foi les manuels sous forme de questions et réponses (Cat. $\mathrm{n}^{\circ} 55,56,57$ et 76 ), les présentations de la matière en tableau synoptique (Cat $n^{\circ} 64,83$ et Ioo) ou les efforts de simplification ou de synthèse (Cat $\left.n^{\circ} 85\right)$. Théoret publia aussi des conférences et plaidoiries, comme cela se faisait souvent à l'époque (Cat. $\mathrm{n}^{0} 80,90$ et IOI).

Théoret ne craignait pas de lancer des projets d'envergure. Alors qu'il éditait déjà le traité de Mignault - dont il assuma la parution des tomes 2 à 6 -il convainquit le juge François Langelier, professeur de droit civil à l'Université Laval, de publier ses notes de cours, ainsi que le rappelait l'auteur dans sa préface: 'L'idée de la publication de mon cours m'était partie de l'esprit depuis longtemps lorsque, il y a deux ou trois ans, $M$. Théoret, le libraire de droit bien connu, me dit 
que, non seulement beaucoup de mes anciens élèves, mais d'autres personnes aussi lui avaient exprimé le désir de le voir publié. ${ }^{\prime 43}$ Initiateur du projet, Théoret mourut cependant avant le lancement du premier tome, dont la paternité revint aux associés Wilson et Lafleur qui venaient d'acquérir l'entreprise.

Dans la mesure du possible, Théoret s'efforça de pénétrer le marché canadien grâce notamment à ses ouvrages de droit commercial et de droit criminel. Il se trouvait ainsi à entrer en concurrence avec la maison Carswell de Toronto qui eut un bureau à Montréal au début du siècle. Par ailleurs, cette compagnie avait lancé quelques ouvrages destinés essentiellement au marché québécois. 44

Certains des auteurs, qui avaient été édités par Périard, demeurèrent fidèles à Théoret. Beauchamp, Holt, Sharp, et Weir revinrent donc dans les champs où ils s'étaient faits une réputation. De nouveaux auteurs furent également recrutés, dont certains firent paraître plusieurs ouvrages: Joseph-Édouard Bédard, MathieuAdolphe Bernard, James Crankshaw, Oscar-Pierre et Albert-Paul Dorais et Rodolphe Lemieux.

Le dynamisme de Théoret, en plus de caractériser ses travaux d'édition, transparaît aussi dans son commerce de librairie. À l'instar de Périard, il publia des catalogues afin de mieux faire connaître ses produits. Il donna cependant une facture spéciale à ses quatre catalogues parus respectivement en I 899 (Cat. ${ }^{\circ}$ I 39), I900 (Cat. $\mathrm{n}^{\circ}$ I4O), I 902 (Cat. $\mathrm{n}^{\circ}$ I4I), et 1905 (Cat. $\mathrm{n}^{\circ}$ I42). Jusqu'alors les catalogues d'ouvrages de droit adoptaient la forme de listes. Théoret apporta une modification substantielle à cette présentation en divisant ses catalogues en deux sections. Le gros des ouvrages disponibles, regroupant des titres importés, continuait d'être énuméré par ordre alphabétique d'auteurs. En revanche, la section consacrée aux ouvrages de droit canadien, donnait une présentation visant à valoriser le contenu d'une bonne part des ouvrages offerts. Les textes étaient empruntés à diverses sourcés. Souvent, il s'agissait d'appréciations louangeuses tirées d'extraits de lettres adressées à l'auteur ou de recensions parues dans des périodiques juridiques ou dans des journaux. Dans quelques cas, faute de réactions externes, le libraire avait simplement reproduit un extrait de la préface. Pour prendre un exemple parmi d'autres, le traité de Mignault fut particulièrement bien servi par les extraits mentionnés au catalogue. Le juge François Langelier concluait ses propos sur l'oeuvre par un jugement non équivoque: 'J'estime donc que cet ouvrage est absolument indispensable aux avocats et aux juges qui ont à étudier des questions de droit civil.'45 
Fort intéressé à faire connaître sa librairie et sa maison d'édition, Camille Théoret participa à l'Exposition universelle de Paris en I900. À cette occasion, fut publié un catalogue - remis vraisemblablement aux visiteurs - qui reprenait la section consacrée aux ouvrages canadiens dans son catalogue paru l'année précédente. Il mérita alors la médaille d'or de la section canadienne dans sa catégorie d'exposants. ${ }^{46}$

À ses premières années dans l'édition, et même par la suite alors qu'il assumait seul la direction de l'entreprise, Théoret semblait parfois éprouver des besoins urgents de liquidité. Des lettres étaient alors envoyées à $\mathrm{McGale}$, qui avait déjà participé au financement de l'achet du fonds de commerce de Périard, lui demandant d'avancer les sommes nécessaires. Des contrats d'édition et une partie de l'inventaire des livres édités par la maison étaient offerts en garantie. ${ }^{47}$ Il est vraisemblable qu'il eût aussi recours au financement bancaire.

Camille Théoret connut une fin tragique. Souffrant depuis plusieurs mois de rhumatisme et d'une neurasthénie aiguë, il se suicida en I 905, alors qu'il n'était âgé que de quarante ans..$^{48}$ Outre son épouse, il laissait deux enfants en bas âge, dont un fils posthume. Peu avant sa mort, Théoret avait fait en sorte d'assurer sa succession, d'une part en accordant une garantie hypothécaire en faveur de son épouse pour garantir une donation à son contrat de mariage ${ }^{49}$ et d'autre part en rédigeant un testament. ${ }^{50}$

En peu de temps, Théoret avait édifié une entreprise florissante, ce qui fut d'ailleurs reconnu à son décès: 'Le défunt, Camille Théoret, était à la tête d'un des établissements de publication des plus prospères au Canada. ${ }^{\prime 5 \mathrm{r}}$ La déclaration présentée par sa veuve au percepteur des revenus sur les successions ${ }^{52}$ tend d'ailleurs à démontrer que les affaires de Théoret furent rapidement rentables. Une dizaine d'années après ses débuts, il bénéficiait de revenus suffisants pour se porter acquéreur de deux immeubles. En 1903, soit l'année suivant son mariage, il devenait propriétaire d'une résidence à Westmount, ainsi que du terrain attenant. L'année suivante, il achetait un édifice de trois étages, situé au 17 rue Saint-Jacques, où il entendait établir son commerce. À son décès les deux immeubles, hypothéqués pour I4 500 \$, étaient évalués 22400 \$. L'inventaire de son fonds de commerce valait $20500 \$$. Compte tenu de l'hypothèque accordée en faveur de son épouse juste avant son décès, la valeur de l'actif de Théoret s'élevait à I9 400 \$à son décès, ce qui à l'époque était une somme respectable, d'autant plus qu'il mourût alors qu'il était encore relativement 
jeune. Théoret parvint, en peu de temps, à faire du commerce exclusif du livre de droit une activité lucrative, ce que n'avait pu faire Amédée Périard avant lui.

\section{Wilson et Lafleur ou la continuité}

Au décès de Camille Théoret, Théophile Lafleur et Wilfrid J. Wilson présentèrent une offre d'achat de l'actif à la succession de Théoret. Elle fut acceptée par les curateurs de la succession avec certaines modifications. ${ }^{53}$

Lafleur gravitait depuis au moins une quinzaine d'années dans le monde de l'édition juridique. Expert lors de l'inventaire après décès des biens de Périard, il travaillait, à la fin des années I 890, comme commis chez Camille Théoret qui lui vouait une grande confiance, le faisant agir comme témoin à certains documents et lui conférant par procuration d'importants pouvoirs de gestion. ${ }^{54} \mathrm{Il}$ était donc la personne toute désignée pour prendre la succession de l'entreprise de son ancien patron.

Wilfrid J. Wilson, d'abord voyageur de commerce, 55 tenait depuis au moins 1897 une librairie spécialisée en droit, aux 4 et 6 de la rue Saint-Jacques. Importateur de livres de droit, il servait d'agent au Québec pour la Canada Law Book de Toronto. Quoiqu'il se présentait comme éditeur juridique, il n'apparaît pas avoir été très actif dans ce secteur. Déloger Théoret et les autres éditeurs, qui sans en faire une spécialité publiaient à l'occasion des ouvrages de droit, n'était certes pas aisé. En fait, nous n'avons retracé que deux ouvrages édités par Wilson avant son association avec Lafleur (tableaux I et 2). Il s'agit d'un catalogue des compagnies canadiennes établi par Philippe Baudouin qui remonte à 1897 (Cat. $\mathrm{n}^{\circ}$ I02) et d'une édition du Code criminel par Charles Lanctot, parue en I9or (Cat. ${ }^{0}{ }^{103}$ ). Même si Wilson transmit son nom à la société, il n'était en fait qu'un prête-nom, puisque c'est son épouse qui détenait le capital et qui s'associa avec Lafleur. ${ }^{56}$

Adèle Martin, l'épouse de Wilson, était la fille d'un meublier de Montréal. ${ }^{57}$ Elle forma avec Lafleur une société pour l'exploitation du commerce acquis de la succession de Théoret. ${ }^{58}$ La société prit le nom de Wilson et Lafleur. L'acte de société prévoyait qu'Adèle Martin se ferait représenter par son mari pour l'exercice de ses droits et l'accomplissement de ses obligations. À titre d'associée, elle apportait un actif supérieur à celui de Lafleur, qui devint son débiteur. L'expérience de ce dernier, le désignait d'emblée respon- 
sable des affaires financières et de la comptabilité de l'entreprise. Assez rapidement, les affaires se gâtèrent entre les associés, Lafleur considérant que Wilson ne fournissait pas un travail suffisant. 59 Cette mésentente amena vraisemblablement, quelques mois plus tard, une restructuration de l'entreprise. À cette occasion, les deux associés se départirent des actifs de la société qui passèrent à une corporation constituée sous le nom de Wilson et Lafleur limitée. En contrepartie, ils reçurent des actions de la nouvelle compagnie. ${ }^{60} \mathrm{La}$ société, qui n'avait alors plus sa raison d'être, fut dissoute. ${ }^{6 \mathrm{I}}$

La charte de la corporation accordait à l'entreprise des pouvoirs très étendus dont, il va sans dire, le droit d'imprimer, d'éditer et de tenir une librairie. La compagnie était entre les mains de cinq actionnaires: Théophile Lafleur et. Adèle Martin, épouse de W.J. Wilson, détenaient respectivement 125 actions, alors que Jean Adolphus Wilson, commerçant, domicilié à Plantagenet en Ontario, Émile Rolland, imprimeur à Montréal, et Joseph Marcelin Wilson, négociant à Montréal, possédaient chacun une action. ${ }^{62}$ Les Wilson avaient donc le contrôle, aussi mince qu'il fût, de la nouvelle compagnie. Ils acceptèrent cependant, de bonne grâce, $d$ 'en confier la présidence à Théophile Lafleur. ${ }^{63}$

L'incorporation $\mathrm{n}^{\prime}$ avait pas permis d'introduire des fonds dans l'entreprise, puisque les deux anciens associés s'étaient départis de leur parts pour une somme égale à la valeur des actions qui leur furent remises. ${ }^{64}$ Le financement de la nouvelle compagnie fut assuré au départ par deux actionnaires minoritaires. J.M. Wilson avança la somme de $3000 \$$, en contrepartie de quoi il reçut des obligations, $^{65}$ tandis que J.A. Wilson acquit, quelques jours après l'incorporation, quarante-sept actions au coût de roo $\$$ chacune, le capital de l'entreprise ayant été augmenté. ${ }^{66}$ Plus tard, l'émission de billets ${ }^{67}$ et même la vente à réméré des Rapports de pratique $e^{68}$ furent des moyens de financement auxquels la compagnie recourut.

Si la mise en place d'une nouvelle structure a pu atténuer quelques temps les récriminations qu'avait manifestées Lafleur à l'encontre $\mathrm{du}$ mandataire de son ancienne associée, les rancoeurs refirent surface rapidement, puisque, en mai I9 I I, il quittait la compagnie et cédait ses actions à J.A. Wilson, qui devint président et à JosephGeorges Laurendeau, avocat réputé, domicilié à Valleyfield. ${ }^{69}$ Quelques mois plus tôt, ce dernier était devenu trésorier de la compagnie après avoir acquis une action symbolique. Laurendeau prit rapidement un rôle prépondérant dans l'administration de la maison. Son arrivée visait certainement à intégrer dans l'entreprise une personne possédant une expertise du monde juridique, puisque aucun 
actionnaire ou employé n'avait de formation en droit.

Après son départ, Théophile Lafleur tint seul une librairie. En novembre I 9I 2, il écrivait à la direction de Wilson et Lafleur afin de leur offrir d'acheter son fonds de commerce et son achalandage. ${ }^{70} \AA$ la suite d'un premier refus, des négociations furent engagées et les administrateurs revinrent sur leur décision et lui proposèrent la somme de $350 \$$, exigeant toutefois qu'une clause de nonconcurrence, empêchant Lafleur de s'occuper désormais du commerce de librairie juridique, apparût au contrat. ${ }^{71}$ Lafleur accepta vraisemblablement ces conditions puisque, par la suite, il ne se manifesta plus dans ce secteur d'activités. Adèle Martin, qui éprouvait des problèmes financiers, fut obligée d'emprunter de l'argent de la compagnie ${ }^{72}$ et se départit, elle aussi, de ses actions au profit de J.A. Wilson, qui devint actionnaire majoritaire, et de Joseph-Georges Laurendeau.

Le personnel de la maison, dans les mois qui suivirent l'incorporation, comptait environ trois employés. Théophile Lafleur, Adèle Martin et J.A. Wilson s'occupaient surtout de la librairie. ${ }^{73} \hat{A}$ l'instar des autres éditeurs-libraires les plus importants de l'époque, la compagnie retint les services d'un voyageur de commerce en la personne de W.J. Wilson, ${ }^{74}$ qui fut cependant rapidement remplacé par C.-E. Gratton. ${ }^{75}$ Contrairement à ce qui prévalait chez Périard et Théoret, la compagnie semblait préférer confier à l'imprimeur le soin de voir à la reliure plutôt que de charger un employé de cette tâche.

Dans les premières années qui suivirent la mise sur pied de la corporation, les administrateurs discutaient entre eux, lors de leur assemblée mensuelle, de tous les projets de quelque importance qui touchaient l'entreprise. Ils échangeaient ainsi sur la possibilité $\mathrm{d}^{\prime}$ acheter la bibliothèque $\mathrm{d}^{\prime}$ une succession ${ }^{76}$ ou de retenir un projet d'édition. ${ }^{77}$ À cet égard, l'opinion de Laurendeau, étant donné ses connaissances du monde juridique, était déterminante. Les propositions au conseil d'administration d'éditer un ouvrage émanaient toujours de lui.

Autant avant qu'après l'incorporation, les activités d'édition d'ouvrages de Wilson et Lafleur accaparaient moins d'énergie qu'elles ne l'avaient fait sous Périard et Théoret. Sous l'association de Wilson et Lafleur (1905-1909), la production annuelle, quoique moins importante (tableau I), ressemblait à celle des dernières années de Théoret (tableau 2). Les monographies (Cat. $\mathrm{n}^{\circ}$ I08, I I I, I 3 et I I4) et manuel (Cat. $n^{\circ}$ I07) constituaient presque la moitié de la production. Un titre retient particulièrement l'attention, 
l'ouvrage de Frederick P. Walton, alors doyen de la Faculté de droit de McGill (Cat. $\mathrm{n}^{0}$ I I4). Ce livre, qui se penchait sur l'étude de la portée et des méthodes d'interprétation du Code civil, tranchait nettement sur le reste de la production éditoriale de l'époque. L'auteur révélait une culture juridique fort étendue et une capacité $\mathrm{d}$ 'analyse scientifique qui se rencontrait peu alors.

Les ouvrages de référence se maintenaient surtout grâce aux travaux de Beauchamp, qui mit à jour son volume sur la jurisprudence du Conseil privé (Cat. $\mathrm{n}^{\circ}$ I04), puis confectionna un nouveau répertoire de jurisprudence, consacré cette fois à la Revue légale (Cat. $\mathrm{n}^{\circ}$ I05). Les codes et textes de loi sont beaucoup moins présents dans la production. Il est vraisemblable que la maison d'édition a pu écouler et même réimprimer les codes sur lesquels Théoret avait des droits, soit ceux d'Oscar-Pierre et Albert-Paul Dorais en langue française et Robert $S$. Weir en langue anglaise. Ceci expliquerait que le code 'Dorais' de 1905 ait été vendu avec une couverture indiquant comme éditeur 'Wilson et Lafleur,' alors que la page titre mentionnait le nom de Théoret. $^{78}$

L'entreprise continua la publication d'ouvrages d'envergure dont la parution était commencée. Deux tomes du traité de Mignault parurent parallèlement aux cinq premiers tomes du traité de Langelier, ces deux projets avaient cependant été initiés par Camille Théoret. Des auteurs qui avaient fait paraître des ouvrages chez Périard et Théoret demeurèrent liés à la nouvelle entreprise, tandis que quelques autres s'ajoutèrent: Edward R. Cameron, Edmond McMahon, Joseph-Fortunat Saint-Cyr, et Joseph Sirois.

La configuration de la production de la maison après l'incorporation changea considérablement. Les ouvrages de consultation prédominèrent nettement (tableau 2). Durant la décennie considérée, pas moins de quinze codes et recueils de lois parurent. Dans le but d'accaparer ce marché, la compagnie obtint même d'une rival, John Lovell \& Son, un engagement à ne pas éditer de Code de procédure civile durant une période de cinq ans. ${ }^{79}$ Les autres catégories de publications, en revanche, perdirent du panache. Elles se limitèrent à deux monographies (Cat. $\mathrm{n}^{0}$ I I 7 et I I 9), à un manuel (Cat. $\mathrm{n}^{0}$ I $3 \mathrm{I}$ ), et à un ouvrage de référence, soit un vaste répertoire portant sur la jurisprudence publiée jusqu'à I9I3, réalisé par JeanJoseph Beauchamp (Cat. $\mathrm{n}^{\circ}$ I I 6). Cette compilation était colossale avec ses quatre volumes.

La production annuelle chuta également (tableau I). Les années de la guerre expliquent en partie cette baisse du nombre de parution, mais il est vraisemblable que même par la suite les crêtes atteintes 
dans les belles années de Théoret ne se reproduisirent plus. Cette diminution des parutions correspond peut-être à un repli de l'édition juridique québécoise qui, autour des années I9ro, semble considérer que son marché est désormais confiné aux seules frontières du Québec.

Wilson et Lafleur éditait deux périodiques qui avaient été acquis avec le fonds de commerce de Théoret soit la Revue légale et de la Revue de jurisprudence. En I9I I, la compagnie devint propriétaire d'une troisième revue: les Rapports de pratique de Québec. Propriété de l'avocat Édouard Fabre Surveyer, les Rapports de pratique étaient un recueil d'arrêts dont la fondation remontait à 1898 . Les parties s'entendirent pour fixer à $3000 \$$ le montant de la transaction, qui en plus de porter sur la propriété du périodique incluait les numéros invendus. Surveyer conservait ses fonctions de rédacteur à raison de $35 \$$ par livraison, soit $425 \$$ par année. Le maintien de trois périodiques juridiques, en plus d'exiger une importante mise de fonds, nécessitait beaucoup de travail. L'éditeur devait voir à la coordination des équipes de rédaction, à l'impression et à l'expédition. La diminution de l'activité éditoriale de l'entreprise durant la seconde décennie du siècle est vraisemblablement attribuable, en partie, à l'emphase que la maison décida de mettre sur l'édition de périodiques plutôt que sur la production de monographies.

Les données sur la rentabilité de l'entreprise sont rares. Il apparaît que sous la direction du tandem Wilson-Laurendeau, elle produisit des revenus appréciables. En I9II, par exemple, les profits s'élevaient à $4722,67 \$, 80$ alors que l'année suivante, ils s'établissaient à $6007,79 \$ .^{81}$

L'entreprise de Wilson et Lafleur continua d'évoluer sous la lancée que Camille Théoret avait donné auparavant à l'édition juridique. Désormais, et pour une longue période, elle occupa presque seule le marché québécois.

\section{Conclusion}

L'imprimé, destiné aux juristes québécois, a été diffusé largement durant la seconde moitié du xIx ${ }^{\mathrm{e}}$ siècle. Cette diffusion $\mathrm{s}^{\prime}$ est $\mathrm{d}^{\prime} \mathrm{abord}$ faite à partir de réseaux déjà constitués. Des éditeurs-libraires et des imprimeurs bien implantés - tels les Beauchemin, Senécal, et Lovell - ouvrirent leurs portes à cette production éditoriale, sans pour autant délaisser leurs autres secteurs.

Dans le dernier quart du siècle cependant le commerce du livre de 
droit acquit son autonomie. Amédée Périard, qui au début des années I 880 ouvrit une librairie spécialisée dans le livre de droit, puis s'adonna à l'édition, jetait ainsi les bases d'une lignée de libraires-éditeurs qui allaient lui succéder.

L'importance prise par l'imprimé juridique durant la seconde moitié du XIX ${ }^{\mathrm{e}}$ siècle doit être analysée dans la perspective plus vaste du développement que connut alors le droit. Jusque là, les ouvrages étrangers avaient suffi aux juristes de la colonie. Toutefois avec la codification du droit civil en $\mathrm{r} 866$, les changements constitutionnels survenus en 1867 et d'autres modifications apportées par la suite, comme l'introduction d'un code criminel en I 892, il devint essentiel d'offrir des ouvrages répondant spécifiquement à cette nouvelle réalité. La professionalisation accrue du droit est aussi un facteur à considérer. La constitution, en I 847 et en I 849, de deux corporations provinciales regroupant avocats et notaires, et la mise sur pied d'un enseignement universitaire du droit, jouèrent sans doute un rôle important dans le développement du commerce du livre de droit.

La 'domestication' de la culture juridique québécoise ${ }^{82}$ serait la principale cause qui expliquerait le développement de l'imprimé juridique au Québec. L'apparition et le maintien d'une entreprise vouée exclusivement au commerce du livre de droit seraient d'ailleurs une preuve qu'un palier a été franchi dans ce processus d'émancipation du droit. ${ }^{83}$ Non seulement la nécessité de développer une production autochtone a-t-elle reçue un accueil favorable, mais elle s'est affirmée comme un secteur spécialisé par rapport au reste de la production éditoriale. ${ }^{84} \mathrm{Il}$ est vraisemblable que c'est justement au tournant du siècle que le droit québécois a pu s'affranchir, pour être perçu comme un droit national. L'émergence du commerce du livre de droit comme secteur spécialisé participerait donc de l'évolution de la culture juridique.

\section{NOTES}

La présente étude a pu être réalisée grâce au soutien financier du Conseil de recherches en sciences humaines du Canada et de la Fondation du Barreau du Québec. L'auteur exprime sa gratitude à monsieur Claude Wilson, président des éditions Wilson et Lafleur ltée, et à madame Christine Lachance, bibliothécaire à la bibliothèque de l'Université Laval, qui lui ont facilité l'accès à un certain nombre de documents. Il remercie également Céline Cyr, de l'Université Laval, Gilles Gallichan, de la Bibliothèque de l'Assemblée 
nationale, et Yvan Lamonde, de l'Université McGill, qui ont bien voulu lire et commenter le manuscrit.

I Parmi les publications récentes, citons: E.C. Surrency, A History of American Law Publishing (New York: Oceana Publications Inc., 1990).

2 Pour une bibliographie, voir: Comité de bibliographie de l'Association québécoise pour l'étude de l'imprimé, Bibliographie des études québécoises sur l'imprimé, 1970-1987 (Montréal: Bibliothèque nationale du Québec, I99I). À titre d'exemple de travaux fournissant des données sur le commerce du livre de droit, voir: Y. Lamonde, 'La librairie Hector Bossange de Montréal (18I 5-1 819) et le commerce international du livre,' dans: C. Galarneau et M. Lemire, éd., Livre et lecture au Québec (I800-1850) (Québec: Institut québécois de recherche sur la culture, I988): 59-92.

3 A. Beaulieu, J.-C. Bonenfant, et J. Hamelin, Répertoire des publications gouvernementales du Québec de 1867-1964/Québec: Imprimeur de la reine, I968); A. Beaulieu, J. Hamelin, et G. Bernier, Répertoire des publications gouvernementales du Québec. Supplément 1965-1968 (Québec: Assemblée nationale, 1970); G. Gallichan, Livre et politique au Bas-Canada, 1792-1849 (Québec: Septentrion, I99I); G. Gallichan, 'Les débats parlementaires du Québec (1792-1964) ou la mémoire des mots,' Cahiers de la Société bibliographique du Canada 27 (1988): 38-79; G. Gallichan, 'L'édition gouvernementale au Québec depuis le XviII ${ }^{\mathrm{e}}$ siècle,' dans: Y. Lamonde, éd., L'édition au Québec; aspects historiques (I 8e-2oe siècles) (Québec: Institut québécois de recherche sur la culture, I983): 269-87; et Y. Thériault, Les publications parlementaires d'hier et d'aujourd'hui (Québec: Assemblée nationale du Québec, I982).

4 À titre d'exemples, citons: G.B. Baker, K.E. Fisher, V. Masciotra, et B. Young, Sources in the Law Library of McGill University for a Reconstruction of the Legal Culture of Quebec, I760-I890 (Montréal: McGill University, 1987); D. Howes, 'La domestication de la pensée juridique québécoise,' Anthropologie et Sociétés I3, I (1989): 103-26; N. Kasirer, 'The Annotated Criminal Code en version québécoise: Signs of Territoriality in Canadian Criminal Law,' Dalhousie Law Journal I 3 (I990): 520-63; R.A. Macdonald, 'Understanding Civil Law Scholarship in Quebec,' Osgoode Hall Law Journal 23 (I985): 573-608.

5 Yvan Lamonde toutefois les signale, tout en se montrant prudent sur leurs dates d'activité (La librairie et l'édition à Montréal, 1776-1920 (Montréal: Bibliothèque nationale du Québec, I991), 95, I 84, I86, et I87).

6 Lovell's Montreal Directory for I88I-82 (Montréal: John Lovell \& Son, I88I), 568 .

7 Lovell's Montreal Directory for I882-83 (Montréal: John Lovell \& Son, I 882$), 478$.

8 L'ouvrage de $\mathrm{Y}$. Lamonde et $\mathrm{D}$. Olivier mentionne plusieurs bibliothèques $\mathrm{d}$ 'hommes de loi qui furent vendues à l'encan (Les bibliothèques personnelles au Québec (Montréal: Bibliothèque nationale du Québec, I983).) Des libraires pouvaient donc tenter de récupérer des lots lors de telles ventes ou 
encore acheter une bibliothèque directement d'un juriste ou de sa succession.

9 Howes, I03, I I I-III5.

Io Pour une liste de catalogues de bibliothèques privées consulter: Y. Lamonde et $\mathrm{D}$. Olivier, Les bibliothèques personnelles au Québec.

II lls ne comprennent pas ici les périodiques voués à la diffusion de la jurisprudence.

I2 E.L. de Bellefeuille, Le Code civil annoté étant le Code civil du Bas-Canada (Montréal: C.O. Beauchemin, I889).

I3 P.-B. Mignault, Code de procédure civile annoté, (. . .) Montréal: J.-M. Valois, I89I).

14 Thomas P. Foran, The Code of Civil Procedure of Lower Canada, [. . .] (Toronto: Carswell, I 886).

I5 Dans une critique parue dans un périodique britannique, l'ouvrage avait été recommandé: 'The work seems to be invaluable to practitioners in the Supreme Colonial Court of Appeal, and on several points, such as the right to extension of patents, will be of use to the general practitioners' ( $A$ Catalogue of Canadian Law Books Arranged Under the Author's Names, Published by $C$. Théoret, Law Bookseller, Importer and Binder also an List of other Canadian Law Publications and Recent English and American Law Works (Montréal: C. Théoret, I899), 7.

I6 $D^{\prime}$ après les inscriptions sur les pages titres des tomes de la revue, Mathieu semble en conserver la propriété jusqu'en 1887 , année où il est désigné rédacteur en chef laissant entendre qu'il agit pour le compte de Périard.

I7 Sur cette bibliothèque, voir: Y. Lamonde, Les bibliothèques de collectivités à Montréal ( $17 e-18$ e siècle) (Montréal: Bibliothèque nationale du Québec, 1979), roo.

I 8 L'édition originale a été éditée à Sorel à l'Atelier typographique de la Revue Légale. La réimpression est probablement postérieure à $\mathrm{I} 887$, année où Périard se portait acquéreur du périodique. D'ailleurs certains des tomes portent l'inscription 'A. Périard, Éditeur-Propriétaire.'

I9 La page titre de la réimpression est marquée de l'inscription 'reprint' et est datée de I 888 .

20 Signalé sous l'intitulé 'Blancs' dans son catalogue de r 883: Catalogue of Law Books, Canadian, French, English and American: Imported and for Sale by A. Périard. Catalogue de livres de droit canadien, français, anglais et américain. (Montréal: A. Périard, r 883), 26.

2 I Inventaire des biens dépendant de la communauté de biens qui a existé entre M. et Dame A. Périard et de la succession de A. Périard, 28 octobre 1892: Greffe du notaire Léandre Bélanger, acte $\mathrm{n}^{\circ} 6170$. D'après cet inventaire, Périard possédait quatre-vingt-sept exemplaires de la thèse de Demers sur les Privilèges sur les biens meubles, I44 de The Jurisprudence of the Privy Council de J.-J. Beauchamp, et 700 du Code civil de Lareau. Le greffe du notaire Léandre Bélanger est conservé au Dépôt des greffes de notaires, au Palais de Justice de Montréal.

22 Catalogue de 1883 , page titre. 
23 Lovell's Montreal Directory for I884-85 (Montréal: John Lovell \& Son, I 884), 545 .

$24 \mathrm{D}^{\prime}$ auprès une mention sur la page titre du tome $\mathrm{I}_{4}$ (1 886) de la Revue légale.

25 Procuration par Amédée Périard à Alfred Turgeon, I4 mai I889, Greffe du notaire Léandre Bélanger, acte $\mathrm{n}^{\circ} 3736$.

26 Procuration de Amédée Périard à Camille Théoret, 22 juillet I89I, Greffe du notaire Léandre Bélanger, acte $\mathrm{n}^{\circ} 5245$.

27 Procuration de Amédée Périard à Théophile Lafleur, 4 juillet I892, Greffe du notaire Léandre Bélanger, acte $\mathrm{n}^{\circ} 5987$.

28 Procuration de Amédée Périard à Louis-Wilfrid Sicotte, I4 mai I 889, Greffe du notaire Léandre Bélanger, acte $\mathrm{n}^{\circ} 3737$.

29 Sur les éditeurs américains, notamment ceux cités ici, voir: Surrency, 2 I I-45.

30 Nouveau catalogue de livres de droit et de jurisprudence de la librairie A. Périard: français-anglais-américain et canadien (Montréal: A. Périard, I89I), première couverture intérieure.

3I Acte de mariage de Amédée Périard et Anne Maher, 28 septembre I 882, Registre de l'état civil de St Mary's Church (Hochelaga) (Archives nationales du Québec à Montréal).

32 La Presse, ro octobre 1892: 4.

33 Périard c. Ville de Montréal, Cour supérieure de Montréal, dossier I8922525 (Archives nationales du Québec à Montréal).

34 'Convocation de créanciers,' La Presse, 9 février 1893: 5.

35 In re A.J. Périard, Cour supérieure, dossier r893-I57 (Palais de Justice de Montréal, Greffe de la Cour supérieure).

36 'Avis de faillite,' La Presse, 25 mars r 893: 4.

37 Vente de fonds de commerce par Charles Desmarteau es-qualité à B.E. McGale, 5 avril I 893, Greffe du notaire Léandre Bélanger, acte $\mathrm{n}^{\circ} 6544$.

38 Vente par B.E. McGale à Whiteford et Théoret, 5 avril I893, Greffe du notaire Léandre Bélanger, acte $\mathrm{n}^{\circ} 6546$.

39 Société entre Camille Théoret et R.A.P. Whiteford, 5 avril I893, Greffe du notaire Léandre Bélanger, acte $\mathrm{n}^{\circ} 6545$.

40 L'ouvrage réimprimé est en tout point semblable à l'édition de $\mathrm{r} 89 \mathrm{I}$. Les deux associés n'ont fait qu'ajouter leur nom sur la page titre, en lieu et place de celui de Périard.

4I Cession de propriété de La Revue de Jurisprudence par Charles-C. de Lorimer Jr. à Whiteford et Théoret, 28 septembre I 895, Greffe du notaire Léandre Bélanger, acte $\mathrm{n}^{\circ}$ 8I 54 .

42 Dissolution de société entre R.A.P. Whiteford et Camille Théoret, 3 octobre I895, Greffe du notaire Léandre Bélanger, acte $\mathrm{n}^{\circ}$ 8I 57 .

43 Cours de droit civil de la province de Québec (Montréal: Wilson \& Lafleur, I905), vii.

44 Outre des éditions du Code civil (voir, supra, note I4), Carswell édita au moins deux ouvrages sur des sujets de droit civil: J.K. Foran, An Essay on Obligations; for lawyers, students and laymen (Toronto: Carswell \& Co., I886) et J.-C. Lamothe, Responsabilité du patron dans les accidents du travail (Montréal: Carswell Co., I905). 
45 Catalogue de livres de droit et de jurisprudence de la Province de Québec (ci-devant Bas-Canada) avec table alphabétique des matières (Montréal: C. Théoret, 1900), 28.

46 A. Dupuis, La province de Québec à l'Exposition de Paris: I900 (Québec: Imprimerie Darveau), I 38.

47 Lettres de Whiteford et Théoret à McGale, ro décembre I 894 et 9 janvier I895, et Lettre de Théoret à McGale, Ier mai I896 (Montréal: Archives de Claude Wilson).

48 'Terrassé par la maladie,' La Presse, 20 mai r905: 24.

49 Garantie hypothécaire par Camille Théoret en faveur de Marie-Yvonne Mongelais, I 5 mars 1905, Greffe du notaire Pierre-Chrysologue Lacasse, acte $\mathrm{n}^{\circ}$ 503I. Le greffe du notaire Pierre-Chrysologue Lacasse est conservé au Dépôt des greffes de notaires, au Palais de Justice de Montréal.

5o Testament de Camille Théoret, 8 avril I905, Greffe du notaire PierreChrysologue Lacasse, acte $\mathrm{n}^{\circ} 5065$.

5 I 'Terrassé par la maladie,' note 48.

52 Déclaration par Madame Yvonne Mongelais pour la succession de Camille Théoret, son mari, au percepteur du revenu sur les successions à Montréal, 20 avril I906, Greffe du notaire Pierre-Chrysologue Lacasse, acte $n^{\circ} 5572$.

53 Il nous a été impossible de retrouver l'acte de vente, toutefois une lettre, datée du 9 septembre I905, envoyée à W.J. Wilson et T. Lafleur par le notaire P.-C. Lacasse, exécuteur testamentaire de Théoret, donne vraisemblablement la substance de l'acte (Montréal: Archives de Claude Wilson).

54 Procuration par Camille Théoret à Théophile Lafleur, 7 juillet I897, Greffe du notaire Léandre Bélanger, acte $\mathrm{n}^{\circ}$ 9410.

55 Lovell's Montreal Directory for 1892-93 (Montréal: John Lovell \& Son, I 892$), 874$.

56 Il s'agit peut-être d'un subterfuge visant à mettre les biens de Wilson, qui était marié en séparation de biens, à l'abri de ses créanciers.

57 Acte de mariage entre Wilfrid Jean Wilson et Adèle Martin, I6 août I886, Registre de l'état civil de la Cathédrale de Montréal (Archives Nationales du Québec à Montréal).

58 Société entre Mme Adèle Martin, épouse de Wilfrid J. Wilson, et Théophile Lafleur, 23 septembre 1905, Greffe du notaire Pierre-Chrysologue Lacasse, acte $\mathrm{n}^{0} 5276$.

59 Mise en demeure par Théophile Lafleur à Mme Adèle Martin et Wilfrid I. Wilson, son mari, I6 janvier 1909, Greffe du notaire Pierre-Chrysologue Lacasse, acte $\mathrm{n}^{\circ} 7 \mathrm{I} 64$.

60 Vente par Wilson et Lafleur à Wilson et Lafleur limitée, I 5 décembre I909, Greffe du notaire Pierre-Chrysologue Lacasse, acte $\mathrm{n}^{\circ} 76 \mathrm{I} 9$.

6I Dépôt par Théophile Lafleur d'une dissolution de société entre lui et Adèle Martin, épouse de Wilfrid J. Wilson, I6 décembre I909, Greffe du notaire Pierre-Chrysologue Lacasse, acte $\mathrm{n}^{\circ} 7620$.

62 Deuxième assemblée des directeurs provisoires, 6 décembre 1909, dans: Wilson et Lafleur ltée, Livre des minutes, tome I: I5-I 6 (Montréal: Archives de Quebecor Inc.). 
63 Première assemblée des directeurs, 6 décembre 1909, dans: Wilson et Lafleur ltée, 23-24.

64 Première assemblée des directeurs, 6 décembre 1909, dans: Wilson et Lafleur ltée, 24 .

65 Première assemblée des actionnaires, 2 décembre 1909, dans: Wilson et Lafleur ltée, I4.

66 Assemblée des directeurs, 29 décembre 1909, dans: Wilson et Lafleur ltée, 25.

67 Assemblée spéciale des directeurs, 4 avril I9I I, dans: Wilson et Lafleur ltée, 46.

68 Assemblée mensuelle des directeurs, I9 octobre I9II, dans: Wilson et Lafleur ltée, 6I.

69 Wilson et Lafleur ltée, Grand livre d'actions. Livre de transferts etc, 2 (Montréal: Archives de Quebecor Inc.). Sur la carrière de Laurendeau, voir: 'L.-Georges Laurendeau,' La Revue du Barreau 7 (1947): 84. Laurendeau représentait le ministère public dans la fameuse affaire Shortis à la fin du siècle: Affaire Shortis (Montréal: C.O. Beauchemin \& Fils, I 896), x.

70 Assemblée spéciale des directeurs, 7 novembre 1912, dans: Wilson et Lafleur ltée, 74 .

7 I Assemblée annuelle des directeurs, 23 janvier I9I3, dans: Wilson et Lafleur ltée, 79 .

72 Assemblée mensuelle des directeurs, 20 juillet 19 I I, dans: Wilson et Lafleur ltée, 57.

73 Assemblée des directeurs, 29 décembre 1909, dans: Wilson et Lafleur ltée, $25-26$.

74 Assemblée spéciale des directeurs, 20 mars et 4 avril I9II, dans: Wilson et Lafleur ltée, 43-47.

75 Assemblée spéciale des directeurs, 4 avril I9I I, dans: Wilson et Lafleur ltée, $46-47$.

76 Assemblée mensuelle des directeurs, 23 février I9I I, dans: Wilson et Lafleur ltée, 39 .

77 Ibid. et Assemblée mensuelle des directeurs, 16 mars I9I I, dans: Wilson et Lafleur ltée, $4 \mathrm{I}$.

78 Exemplaire signalé dans Bibliographie du Québec, I82I-1967, tome 20 (Montréal: Bibliothèque nationale du Québec, 1988), 65, no 20_0493 et conservé à la Bibliothèque nationale du Québec.

79 Assemblée des directeurs, 28 janvier I9I 5, dans: Wilson et Lafleur ltée, 89.

80 Assemblée annuelle des actionnaires, 25 janvier 1912, dans: Wilson et Lafleur ltée, 67.

8I Assemblée annuelle des actionnaires, 23 janvier 1913, dans: Wilson et Lafleur ltée, 75-76.

82 Sur ce concept, voir: Howes.

83 Aux États-Unis, le secteur de l'édition juridique semble avoir connu un développement important au lendemain de la période coloniale (Surrency, 3-4).

84 L'édition musicale connut un cheminement similaire, voir: M. Calderisi, 
L'édition musicale au Canada, I800-1867 (Ottawa: Bibliothèque nationale du Canada, I98r).

TABLEAU I

Production des éditeurs par année

\begin{tabular}{|c|c|c|c|c|c|}
\hline \multicolumn{2}{|c|}{ A. PÉRIARD } & \multicolumn{2}{c|}{$\begin{array}{c}\text { WHITEFORD } \\
\text { ET THÉORET }\end{array}$} & \multicolumn{2}{c|}{ C. THÉORET } \\
\hline ANNÉE & NOMBRE & ANNÉE & NOMBRE & ANNÉE & NOMBRE \\
\hline 1884 & 4 & 1894 & 4 & 1895 & 1 \\
\hline 1885 & 4 & 1895 & 3 & 1896 & 4 \\
\hline 1886 & 4 & & & 1897 & 9 \\
\hline 1887 & 4 & & & 1898 & 7 \\
\hline 1888 & 4 & & & 1899 & 6 \\
\hline 1889 & 10 & & & 1900 & 3 \\
\hline 1890 & 6 & & & 1901 & 8 \\
\hline 1891 & 5 & & & 1902 & 5 \\
\hline 1892 & 0 & & & 1903 & 5 \\
\hline & & & & 1904 & 1 \\
\hline & & & & 1905 & 4 \\
\hline
\end{tabular}

\begin{tabular}{|c|c|c|c|c|c|}
\hline \multicolumn{2}{|c|}{ W.J. WILSON } & \multicolumn{2}{c|}{$\begin{array}{c}\text { WILSON ET } \\
\text { LAFLEUR ASS. }\end{array}$} & \multicolumn{2}{c|}{$\begin{array}{c}\text { WILSON ET } \\
\text { LAFLEUR LTÉE }\end{array}$} \\
\hline ANNÉE & NOMBRE & ANNÉE & NOMBRE & ANNÉE & NOMBRE \\
\hline 1897 & 1 & 1905 & 3 & 1910 & 3 \\
\hline 1898 & 0 & 1906 & 1 & 1911 & 1 \\
\hline 1899 & 0 & 1907 & 4 & 1912 & 2 \\
\hline 1900 & 0 & 1908 & 1 & 1913 & 2 \\
\hline 1901 & 1 & 1909 & 2 & 1914 & 1 \\
\hline & & & & 1915 & 1 \\
\hline & & & & 1916 & 1 \\
\hline & & & & 1917 & 1 \\
\hline & & & & 1918 & 1 \\
\hline & & & & 1919 & 3 \\
\hline & & & & 1920 & 3 \\
\hline
\end{tabular}

Ce tableau a été réalisé à partir de l'inventaire qui se retrouve en annexe. 
TABLEAU 2

Production des éditeurs par catégories d'ouvrages

\begin{tabular}{|c|c|c|c|c|}
\hline ÉdIteurs & TRAITÉs & $\begin{array}{c}\text { MONO- } \\
\text { GRAPHIES }\end{array}$ & MANUELs & $\begin{array}{c}\text { OUVRAGES de } \\
\text { RÉFERENCE }\end{array}$ \\
\hline A. Périard & & 14 & & 5 \\
\hline Whiteford et Théoret & 1 & 2 & & 2 \\
\hline C. Théoret & & 15 & 8 & 3 \\
\hline W.J. Wilson & & & & 1 \\
\hline Wilson et Lafleur ass. & 1 & 4 & 1 & 3 \\
\hline Wilson et Lafleur ltée & & 2 & 1 & 1 \\
\hline
\end{tabular}

\begin{tabular}{|c|c|c|c|c|}
\hline ÉDIteurs & $\begin{array}{c}\text { RECUEILS } \\
\text { d'ARRÉTS }\end{array}$ & $\begin{array}{c}\text { CODES ET } \\
\text { LOIS }\end{array}$ & AUTRES & TOTAL \\
\hline A. Périard & 3 & 18 & 1 & 41 \\
\hline Whiteford et Théoret & & 2 & & 7 \\
\hline C. Théoret & & 24 & 3 & 53 \\
\hline W.J. Wilson & & 1 & & 2 \\
\hline Wilson et Lafleur ass. & & 2 & & 11 \\
\hline Wilson et Lafleur ltée & & 15 & & 19 \\
\hline
\end{tabular}

Ce tableau a été réalisé à partir de l'inventaire qui se retrouve en annexe. 


\section{Annexe}

\section{Catalogue de la production éditoriale}

Ce catalogue a été dressé à l'aide du fichier informatisé de la Bibliothèque de l'Université Laval, de Bibliographie du Québec, I82I-1967, tomes I-23 (Montréal: Bibliothèque nationale du Québec, I980-I 99I) et des banques de données de la Bibliothèque nationale du Canada et de la Bibliothèque nationale du Québec.

SIGNIFICATION DES SIGLES DE LOCALISATION

ICMH: Institut canadien de microreproductions historiques

OONL: Bibliothèque nationale du Canada, Ottawa

otyL: Bibliothèque de la Faculté de droit (Osgoode Hall)

de l'Université York, Toronto

Qмвм: Bibliothèque de la Ville de Montréal, Montréal

QMBN: Bibliothèque nationale du Québec, Montréal

QMM: Bibliothèque de la Faculté de droit de l'Université McGill, Montréal

QMuM: Bibliothèque de la Faculté de droit de l'Université de Montréal, Montréal

QQLA: Bibliothèque de l'Université Laval, Québec

A. Ouvrages édités par Amédée Périard (1 884-I 89I)

I. ARChambault, J.L. (Joseph-Louis).

Le barreau canadien au Conseil privé: extraits d'une conférence prononcée le I4 mars 1889 devant le Club conservateur de Montréal. Montréal: A. Périard, I889. I2 p.

II Loc.: ICMH, no 2197; OONL; QMBN

2. BEAUCHAMP, J.J. (Jean-Joseph).

The jurisprudence of the Privy Council containing a digest of all the decisions of the Privy Council; a sketch of its history; notes on the constitution of the judicial committee; a summary of its procedure; and also three appendices. Montréal: A. Périard, I 891 . xiv, 920 p.

II Loc.: QQLA

Cet ouvrage fut réimprimé en I 894 ou I 895 par les éditeurs Whiteford et Théoret 
3. BORTHWICK, J. Douglas (John Douglas).

History of the Montreal prison from A.D. 1784 to A.D. I886, containing a complete record of the troubles of $1837-1838$, burning of the Parliament Buildings, in I849, the St. Alban's Raiders, I864, the two Fenian Raids of I 866 and I870, and a chronological digest of all the principal events for the past hundred years, valuable statistical tables from the police and Recorder's Courts, curious proclamation, warrants and other documents never before printed, relating to the Patriots of ' 37 , and the administration of justice from the commencement of the courts in 1784 . Montréal: Périard, I886. vii, $269 \mathrm{p}$.

II Loc.: ICMH, no I 86; QMBN

4. [Code civil, Code de procédure civile (1 890). Amendements. Anglais] Amendments to the Civil Code and to the Code of Civil Procedure (53 and 54 Vic. I890.). Montréal: A. Périard, I891. I6 p.

II Loc.: QQLA

5. Cushing, Charles.

Cushing's notarial form book with a treatise or historical outline of the notarial profession. Montréal: A. Périard, r 887 . xxiii, 260 p.: formules.

II Loc.: ICMH, no 6I82; QMBN

6. DANDURAND, Raoul; LANCTOT, Charles.

Traité théorique et pratique de droit criminel comprenant la nature, les caractères essentiels et la division des crimes, les règles relatives à la responsabilité pénale et à la complicité, les offenses statutaires et de droit commun, la procédure régulière et la procédure sommaire avec renvois aux statuts et aux ouvrages des criminalistes canadiens, anglais et américains. Montréal: A. Périard, I 890 . xxii, $695 \mathrm{p}$.

II Loc.: ICMH, no I0568; QMBN; QQLA

7. DEMERS, Philippe.

Des privilèges sur les biens meubles: thèse pour le doctorat présentée et soutenue le I 2 janvier I 889. Montréal: A. Périard, I 889. $\mathrm{ix}$, Irg $\mathrm{p}$.

II Loc.: ICMH, no 7897; QMBN; QQLA

8. DUBREUIL, J.F. (Joseph-Féréol).

The reference book being a detailed index of all public and private statutes and orders in council contained therein passed by the Canadian Parliament and by the Legislatures of the several Canadian Provinces, since Confederation, down to and including the year I 887. 2e éd. Montréal: A. Périard, I888. vii, 408 p.

II Loc.: ICMH, no I0574; QMBN; QQLA

9. FRÉMONT, J. (Joseph).

Compendium of Dominion laws of Canada, I867-1883, in force on the first day of January, I 884, indicating amendments, repeals, 4 c., with index. Montréal: A. Périard, I884. 2 I I p. 
II Loc.: ICMH, no 3254; QMBN; QQLA

Io. HOLT, Chas. M. (Charles Macpherson).

Principles of Canadian railway law with the Canadian jurisprudence and the leading english and american cases, to which is added the Dominion Railway Act as amended up to I886: with references to the provincial statutes of Ontario and Quebec, forms of proceedings in expropriation, and a complete index. Montréal:

A. Périard, I 886. xix, $256 \mathrm{p}$.

II Loc.: ICMH, no I0620; QQLA

II. LAREAU, Edmond.

[Code civil (1885)]

Le Code civil du Bas-Canada contenant sous chaque article les amendements et autres dispositions législatives qui affectent le texte; l'indication des autorités citées par les codificateurs et d'autres plus récentes; la citation des arrêts des tribunaux de la province de Québec et suivi d'une table des matières et d'une table de concordance avec le Code Napoléon et le Code de commerce français. Montréal: A. Périard, I885. xviii, $666 \mathrm{p}$.

II Loc.: ICMH, no I0894; QMBN; QMM; QQLA

I2. LAREAU, Edmond.

[Code civil (r888)]

Code civil du Bas-Canada contenant sous chaque article les amendements et autres dispositions législatives qui affectent le texte, jusqu'au rer janvier I 888, l'indication des autorités citées par les codificateurs et d'autres plus récents; la citation des arrêts des tribunaux de la province de Québec. Montréal: A. Périard, I888. xviii, $656 \mathrm{p}$.

II Loc.: ICMH, no I0822; QMBN

I3. LAREAU, Edmond.

Histoire du droit canadien depuis les origines de la colonie jusqu'à nos jours. Tome I: Domination française. Montréal: A. Périard, I888. $\mathrm{X}, 5 \mathrm{I} 8$.

II Loc.: ICMH, no I 2249; QQLA

I4. LAREAU, Edmond.

Histoire du droit canadien depuis les origines de la colonie jusqu'à nos jours. Tome II: Domination anglaise. Montréal: A. Périard, I 889. $544 \mathrm{p}$.

II LOC.: ICMH, nO I 2250; QQLA

I 5. LORANGER, T.J.J. (Thomas-Jean-Jacques).

Lettres sur l'interprétation de la constitution fédérale dite l'Acte de l'Amérique britannique du Nord, I867: deuxième et troisième lettres. Montréal: A. Périard, I884. P. 64-126.

II Loc.: ICMH no I0674; QMBN

I6. LORRAIN, Léon.

[Code civil, Code de procédure civile, Code municipal (I890)]

Les Codes de la province de Québec mis au courant de la 
législation: Code civil, Code de procédure civile, Code municipal. Montréal: A. Périard, I890. 417, 324, 220 p.: formules.

II Loc.: QMBN

17. LORRAIN, Léon.

[Code de procédure civile (I 886)]

Code de procédure civile mis au courant de la législation offrant sous chaque article la conférence des articles, et une référence aux autorités citées par les codificateurs, ainsi qu'aux décisions judiciaires suivi d'un supplément contenant entre autres choses les règles de pratique, les tarifs d'honoraires des avocats, etc., ainsi que des régistrateurs et des notaires, etc., d'un petit formulaire et d'une table alphabétique. Montréal: A. Périard, 1886. xxiv, 626 p.: formules.

II Loc.: QMBN; QMM

I8. LORRAIN, Léon.

[Code de procédure civile (I889)]

Les Codes de la province de Québec mis au courant de la législation: Code civil, Code de procédure civile, Code municipal: Code de procédure. Montréal: A. Périard, 1889. 328 p.: formules.

II Loc.: QMBN

19. LORRAIN, Léon.

[Code municipal (1889)]

Les Codes de la province de Québec mis au courant de la législation: Code civil, Code de procédure civile, Code municipal: Code municipal. Montréal: A. Périard, I889. 220 p.: formules.

II Loc.: QMBN

20. LORRAIN, Léon.

[Lois]

Code des locateurs et locataires ou Traité complet du bail à loyer, du bail à ferme, du bail de meubles, du bail à cheptel et du bail emphytéotique d'après le Code civil, la doctrine des auteurs et la jurisprudence des tribunaux de la province de Québec suivi de formules de baux et autres actes sous seing privé relatifs au louage. Montréal: A. Périard, I885. xv, 353 p.: formules.

II Loc.: ICMH, no I0702; QMBN; QQLA

2I. LORRAIN, Léon.

Précis de la procédure sommaire spéciale dans les causes entre locateurs et locataires suivi de formules d'actions. Montréal: A. Périard, $1884.48 \mathrm{p}$.

II Loc.: ICMH, no 2452 I

22. MAJOR, Alfred B.

Legal sketches. Montréal: A. Périard, I 887. 147 p.

II Loc.: ICMH, no I0690; QMBN

23. MARChand, F.G. (Félix-Gabriel).

Manuel et formulaire général et complet du notariat de la province de Québec contenant $I^{\circ} 1^{\prime}$ histoire du notariat; $2^{\circ} 1^{\prime}$ 'organisation 
actuelle du notariat dans la province de Québec; $3^{\circ}$ un traité sur la responsabilité civile des notaires; $4^{\circ}$ un formulaire français-anglais des actes des notaires; $5^{\circ}$ les tarifs des notaires, des régistrateurs, et des extraits des tarifs judiciaires suivi d'index alphabétiques des formules, en français et en anglais. Montréal: A. Périard, I891. 584 p.: formules.

II Loc.: ICMH, no 9892; QQLA

Cet ouvrage fut réimprimé en 1892 .

24. Mathieu, Michel.

[Code municipal (1 886)]

Code municipal de la province de Québec, mis au courant de la législation et de la jurisprudence, suivi d'un appendice comprenant des extraits des statuts concernant les corporations municipales et leurs officiers, et relatifs aux lois de l'instruction publique, aux élections parlementaires, à l'admission des aliénés dans les asiles, aux licences et à la division territoriale de la province de Québec. Montréal: A. Périard, I886. xiv, 406 p.

II Loc.: ICMH, no 36300; OONL; QMBN; QQLA

Cet ouvrage fut réimprimé en 1887 .

25. Mathieu, Michel.

[Code municipal ( 1887 ). Anglais]

Municipal Code of the province of Quebec, with notes of all the amendments thereto, and decisions thereon, and an appendix containing extracts from the statutes, relating to municipal corporations and their officers, public education, parliamentary elections, lunatic asylums, licences and the territorial division of the province. Montréal: A. Périard, 1887 . xiv, 388 p.

II LOc.: ICMH, no IOI 55; QMBN

26. MCGIBBON, R.D.

The law of abandonment of property and assignments, in the province of Quebec with forms. Montréal: A. Périard, I885. vi, 45 p.: formules.

II Loc.: ICMH, no 4870

27. Mignault, P.B. (Pierre-Basile).

Manuel de droit parlementaire ou cours élémentaire de droit constitutionnel précédé d'une esquisse historique du régime parlementaire en Angleterre et au Canada. Montréal: A. Périard, I 889. $\mathrm{xxi}, 476 \mathrm{p}$.

II Loc.: ICMH, no I0074; QQLA

28. PELISSIER, L.E. (Louis-Ernest).

Traité théorique et pratique de la responsabilité des architectes et des entrepreneurs contenant l'exposé raisonné des règles générales et spéciales ayant rapport au louage d'ouvrage et auxquelles sont assujettis les architectes, les entrepreneurs et les ingénieurs-civils, suivi d'un appendice contenant les règles concernant la construction des maisons à Montréal. Montréal: A. Périard, I89i. xv, 208 p. 
II LOc.: QMBN; QQLA

29. RAMSAY, Thomas Kennedy.

Montreal condensed reports. Précis des décisions des tribunaux du district de Montréal. I854. 2e éd. Montréal: A. Périard, 1884. xiv, I $43 \mathrm{p}$.

II Loc.: QQLA

30. RAMSAY, Thomas Kennedy.

Ramsay's appeal cases with notes and definitions of the civil and criminal law of the province of Quebec including a large number of decisions in appeal otherwise unreported and brought down to the beginning of the year 1887 to which is appended a list of all the cases carried to the Supreme Court and Privy Council with the text of the judgments in the Privy Council. Édite par Charles Henry Stephens. Montréal: A. Périard, I887. vi, ro63 p.

II Loc.: ICMH, no I 2302; QMBN

31. Rapports des jugements rendus en Cour supérieure à Montréal et dans la Cour du banc de la reine (en appel) sur la constitutionnalité de l'Acte imposant des taxes sur les banques et les corporations commerciales. Montréal: A. Périard, I885. iv, 202 p.

II LOC.: QQLA

32. SAUVAlle, P.M. (Marc).

Manuel des assemblées délibérantes: guide des présidents, viceprésidents, secrétaires et membres d'assemblées. Montréal: A. Périard, I890. viii, I77, xii p.

II Loc.: ICMH, no I 3099; QQLA

33. SHARP, William Prescott.

[Code civil (r 889). Anglais]

Civil Code of Lower Canada with the amendments effected by imperial, federal and provincial legislation and all reported cases from the earliest reports up to Ist October I888. Montréal: A. Périard, I 889. 2 vol. (xxvii, 746; 826 p.): formules.

II Loc.: QMBN; QQLA

34. SHARP, William Prescott.

[Code civil ( I 889). Supplément. Anglais]

Supplement no I to Sharp's Civil Code, containing all statutory enactments and a digest of all reported cases affecting the Civil Code of Lower Canada from the Ist October I888 to Ist October I889. Montréal: A. Périard, I889. 106 p.

II Loc.: QMBN

35. ShARP, William Prescott

[Code civil ( 1890 ). Supplément. Anglais]

Supplement no 2 to Sharp's Civil Code, containing all statutory enactments and a digest of all reported cases affecting the Civil Code of Lower Canada from the Ist October I889 to Ist October I890. Montréal: A. Périard, I890. I 32 p.

II Loc.: QMBN 
36. Thévenot d'essaule de SAvigny, Claude François

Traité des substitutions fidéicommissaires contenant toutes les connaissances essentielles selon le droit romain et le droit français avec des notes sur l'ordonnance de 1747 . Édité par Michel Mathieu. Montréal: A. Périard, I888. xxix, 527 p.

II Loc.: ICMH, no 24697; QQLA

37. WEIR, Robert Stanley

An insolvency manual containing the articles of the Code of Civil Procedure relating to abandonment of property, capias ad respondendum, attachments before judgment and revendication together with notes upon conservatory attachment, compiled and collated with the most recent amendments and the latest decisions of the courts. Montréal: A. Périard, I890. x, 90 p.: formules.

II Loc.: ICMH, no 10750

38. WEIR, Robert Stanley.

[Loi sur les lettres de change, (r89I)]

The Bills of Exchange Act, I890, being an Act relating to bills of exchange, cheques and promissory notes (53 Victoria, Chapter 33 of the acts of the Parliament of Canada) together with an introduction, explanatory notes and an index. Montréal: A. Périard, I89r. $\mathrm{xii}, 88 \mathrm{p}$.

II Loc.: ICMH, no 10749

39. WEIR, W.A. (William Alexander).

[Code civil, Code de procédure civile, Code municipal (I 890). Anglais]

Codes of the province of Quebec as amended to I890: Civil Code, Code of Civil Procedure, Municipal Code. Montréal: A. Périard, I 890.446 , I6, viii, 338, 220 p.: formules.

II Loc.: QMM

40. WeIr, W.A. (William Alexander).

[Code de procédure civile (I 889). Anglais]

Code of Civil Procedure of the Province of Quebec with amendments collated and incorporated. Montréal: A. Périard, I889. viii, 338 p.: formules.

II Loc.: QMBN; QMM

4I. WEIR, W.A. (William Alexander).

[Code municipal ( 1889 ). Anglais]

Municipal Code of the province of Quebec, and index with amendments collated and incorporated. Montréal: A. Périard, I889. ix, $220 \mathrm{p}$.

II Loc.: QMBN 


\section{B. Ouvrages édités par Whiteford et Théoret (1 894-I 895)}

42. AUGER, J.C. (Joseph-Cyrille).

Informations précises et officielles touchant l'enregistrement et l'impôt contenant les instructions aux régistrateurs, les noms et résidences des régistrateurs, les cadastres actuellement promulgués et l'analyse des interprétations officielles sur l'impôt. Montréal: Whiteford et Théoret, $\mathrm{I} 895.45 \mathrm{p}$.

II Loc.: ICMH, no 2447; OONL; QMBN

43. BEAUChamp, J.J. (Jean-Joseph).

Le répertoire de la Revue légale sous forme alphabétique et chronologique contenant un résumé des décisions canadiennes et étrangères qui y ont été publiées, avecles noms de la Cour, des juges et des parties, la date du jugement et les autorités citées, ainsi qu'une référence aux articles de fond qui s'y trouvent, suivi d'une table générale des causes. Montréal: Whiteford et Théoret, I894. xxvii, I $179 \mathrm{p}$.

II Loc.: ICMH, no 3336; QMBN; QQLA

44. CRANKSHAW, James.

A practical guide to police magistrates and justices of the peace with an alphabetical synopsis of the criminal law and an analytical index. Montréal: Whiteford et Théoret, r895. xxx, v, 707 p.: formules.

II Loc.: ICMH, no 5 I2, OONL; QMBN; QQLA

45. CRANKSHAW, James.

[Code criminel (I 894). Anglais]

The Criminal Code of Canada and the Canada Evidence Act, I893, with an extra appendix containing the Extradition Act, the extradition convention with the United States, the Fugitive Offenders' Act, and the House of Commons debates on the code and an analytical index. Montréal: Whiteford et Théoret, I 894. lxxxviii, 976 p.: formules.

II Loc.: QMBN; QQLA

46. DORION, C.É. (Charles-Édouard).

De l'admissibilité de la preuve par témoins en droit civil: thèse pour le doctorat. Montréal: Whiteford et Théoret, I894. I59 p.

II LOc.: OONL; QMBN; QQLA

47. Mathieu, M. (Michel).

[Code municipal (I 894)]

Code municipal de la province de Québec mis au courant de la législation et de la jurisprudence, suivi d'un appendice comprenant des extraits des statuts concernant les corporations municipales et leurs officiers, et relatifs aux lois de l'instruction publique, aux élections parlementaires, à l'Acte des licences de Québec, le tarif des régistrateurs et des notaires et l'Acte du cens électoral du 
Canada. Montréal: Whiteford et Théoret, I894. xxxvi, 639 p.: formules.

II Loc.: ICMH, no IOI 54; QMBN

48. Mignault, P.B. (Pierre-Basile).

Le droit civil canadien basé sur les 'Répétitions écrites sur le code civil' de Frédéric Mourlon avec revue de la jurisprudence de nos tribunaux. Montréal: Whiteford et Théoret, I895-1916. 9 tomes.

II Loc.: ICMH, no I0079-IOO88; QMBN; QQLA

Le premier tome de ce traité fut réimprimé par $\mathrm{C}$. Théoret.

\section{Ouvrages édités par Camille Théoret (1895-1905)}

49. Аввотт, Harry.

A treatise on the railway law of Canada. Embracing constitutional law. - The law of corporations. - Railway securities. - Eminent Domain. - Contracts. - Common carriers. - Negligence. - Damages. - Master and servant. - Text of Dominion and provincial railway acts, etc. - Forms of proceedings in expropriation. Montréal: C. Théoret, I896. xxxiv, $684 \mathrm{p}$.

II Loc.: ICMH, no I0500; QMBN; QQLA

50. BEAUCHAMP, J.J. (Jean-Joseph).

[Code civil (1904-1905). Français et anglais]

Le code civil de la province de Québec annoté contenant les textes français et anglais et celui du Code Napoléon, les autorités et les remarques des codificateurs, le droit ancien, la concordance des articles, le droit statutaire, la doctrine canadienne, la jurisprudence canadienne, la doctrine française et les règles de droit avec divers appendices. Montréal: C. Théoret, 1904-1905. 3 t. en 2 v. (xiv, I 83 ; vi, I 687 p.)

II Loc.: QMBN; QQLA

5I. BÉDARD, J.-É. (Joseph-Édouard).

[Code municipal (I 898). Français et anglais]

Code municipal de la province de Québec annoté, texte français et anglais, mis au courant de la législation et de la jurisprudence suivi des statuts concernant les corporations municipales et leurs officiers, relatifs aux élections parlementaires, aux licences, aux jurés et jurys, etc., etc.. Montréal: C. Théoret, r898. xxv, 663 p.: formules.

II Loc.: ICMH, no I0527; QMBN; QQLA

52. BÉDARD, J.-É. (Joseph-Édouard).

[Code municipal (1902). Français et anglais]

Code municipal de la province de Québec annoté I898-I902 suivi d'un supplément qui le met au courant de la législation et de la 
jurisprudence jusqu'au rer juillet 1902 et contient les dispositions statutaires concernant les officiers munidipaux (sic) quant aux élections parlementaires, licences et jurés. Montréal: C. Théoret, I902. $47 \mathrm{I}$, [I 52] p.: formules.

II Loc.: QMBN

53. BÉDARD, J.-É. (Joseph-Édouard).

[Code municipal (I902). Supplément. Français et anglais]

Supplément au Code municipal de la province de Québec annoté mis au courant de la législation et de la jurisprudence jusqu'au rer mai 1902 et suivi d'extraits des statuts concernant les corporations municipales et leurs officiers, relatifs aux élections parlementaires, aux licences, aux jurés et jurys, etc., etc.. Montréal: C. Théoret, I902. I23, ix p.

II Loc.: QMBN

54. BÉDARD, J.-É (Joseph-Édouard).

[Code municipal (1905)]

Code municipal de la province de Québec annoté mis au courant de la législation et de la jurisprudence, et suivi des dispositions statutaires concernant les officiers municipaux quant aux élections parlementaires, licences, jurés, $\Theta c .2 \mathrm{e}$ éd. Montréal: C. Théoret, I905. xxix, 715 p.: formules.

II Loc.: QMBN

55. Bernard, Mathieu A. (Mathieu-Adolphe).

Manuel de droit commercial théorique et pratique de la province de Québec. Montréal: C. Théoret, I90I. I34 p.

II Loc.: QMBN; QQLA

56. Bernard, Mathieu A. (Mathieu-Adolphe).

Manuel de droit constitutionnel et administratif comprenant la constitution anglaise, le gouvernement de la Puissance du Canada, les gouvernements provinciaux, le système municipal et l'éducation dans les provinces, précédé de l'histoire politique du Canada et suivi de 'T'Acte de l'Amérique britannique du Nord, 1867' et ses amendements. Montréal: C. Théoret, 1901. vi, $222 \mathrm{p}$.

II Loc.: QMBN

57. BerNard, Mathieu A. (Mathieu-Adolphe).

Manuel de droit international, public et privé: ouvrage basé sur le droit international de Charles Calvo et contenant les dispositions du Code civil de la province de Québec et des statuts impériaux et fédéraux applicables à la matière. Montréal: $\mathrm{C}$. Théoret, I90I. xxix, $256 \mathrm{p}$.

II Loc.: QMBN

58. BLIGH, Harris H. (Harris Harding).

The Quebec law index embracing all the legislation of the province of Quebec from 1867, down to and including the year 1898 . Montréal: C. Théoret, $1898.283 \mathrm{p}$.

II Loc.: ICMH, no I47; QQLA 
59. BRUNET, Ludovic.

De l'Habeas corpus ad subjiciendum en matière criminelle et civile. Montréal: C. Théoret, 1901. I53 p.

II LOC.: QMBN; QQLA

6o. CANADA.

[Acte des compagnies (I 902)]

The Canada Companies' Act I902. Annotated by Morris, Holt \& Gaudet, solicitors, Montreal, at the request of the Canadian Bankers Association. Montréal: C. Théoret, 1902. $42 \mathrm{p}$.

II Loc.: QMBN

61. CAzEs, Paul de.

[Code scolaire (1899)]

Code scolaire de la province de Québec contenant la Loi de l'instruction publique et un grand nombre de décisions judiciaires s'y rapportant, les règlements scolaires du Comité catholique du Conseil de l'instruction publique, des tables de concordance des articles de la Loi de l'instruction publique à ceux des Statuts refondus de Québec, et des articles des statuts refondus à ceux de la Loi de l'instruction publique, la liste des jugements cités et une table des abréviations. Ière éd. Montréal: C. Théoret, I899. xxxix, 279, I00 p.: formules.

II Loc.: ICMH, no I0549; QMBN

62. Cousineau, Philémon.

Des corporations: thèse pour le doctorat présentée et soutenue le 8 avril r90I. Montréal: C. Théoret, I90I. xii, I02 p.

II Loc.: QMBN

63. CRANKSHAW, James.

A practical guide to police magistrates and justices of the peace, with an alphabetical synopsis of the criminal law of Canada and an analytical index. 2e éd. Montréal: C. Théoret, I $905 . x x x i v, v, 845$ p.: formules.

II Loc.: QMBN

64. CRANKSHAW, James.

An analytical synopsis of the Criminal Code and of the Canada Evidence Act. Montréal: C. Théoret, I899. vii, I36 p.

II Loc.: ICMH, no 96; OONL; QQLA; QMBN

65. CRANKSHAW, James.

[Code criminel (I902). Anglais]

The Criminal Code of Canada and the Canada Evidence Act, with their amendments, including the amending acts of 1900 and I9OI, and extra appendices, containing the Imperial Criminal Evidence Act, the Foreign Enlistment Act, the Canadian Interpretation Act Amendment Act, the Victoria Day Act, the Demise of the Crown Acts, the Alien Labor Act, the Yukon Territory Acts, the Canadian Fugitive Offenders' Act and Extradition Acts, the extradition convention with the United States, and a list of extradition treaties, 
etc.. 2e éd. Montréal: C. Théoret, I 902. cvi, I 62 p.: formules.

II LOc.: QMBN; QQLA

66. cusson, Victor.

Codes des huissiers et des shérifs de la province de Québec contenant les textes anglais et français; la jurisprudence complète jusqu'à ce jour, et des commentaires. Montréal: C. Théoret, I899. viii, 2 I 3 p.

II Loc.: ICMH, no I0566

67. DORAIS, O.P. (Oscar-Pierre); DORAIs, A.P. (Albert-Paul).

[Code civil (I 897)]

Code civil de la province de Québec, mis au courant de la législation jusqu'au premier octobre I 897, comprenant la mention des différentes lois qui l'ont modifié, une comparaison ou conférence de ses articles entre eux et avec ceux du Code de procédure, des renvois aux statuts qui s'y rapportent, l'Acte fédéral des lettres de change, I890, tel qu'amendé, et un index alphabétique. Montréal: C. Théoret, I897. xxv, 485, li p.: formules.

II LOc.: ICMH, no I 2552; OONL; QMBN; QQLA

68. DORAIS, O.P. (Oscar-Pierre); DORAIs, A.P. (Albert-Paul).

[Code civil (I905)]

Le Code civil de la province de Québec collationné sur le texte officiel et mis au courant de la législation, comprenant la mention des différentes lois qui l'ont modifié, une comparaison ou conférence de ses articles entre eux et avec ceux du Code de procédure, des renvois aux statuts qui s'y rapportent, la mention des articles correspondants du Code Napoléon, l'Acte fédéral des lettres de change, 1890, tel qu'amendé, et un index alphabétique. 2e éd. Montréal: C. Théoret, I905. xxvii, 684 p.: formules.

II Loc.: QMBN; QQLA

69. DORAIS, O.P. (Oscar-Pierre); DORAIs, A.P. (Albert-Paul).

[Code de procédure civile (I 897)]

Code de procédure civile de la province de Québec en vigueur le premier septembre I897, comprenant les observations spéciales des commissaires chargés de la révision et modification du Code de procédure civile du Bas-Canada, une table de concordance des articles entre le rapport des commissaires et le code, les lois et autorités qui ont été la base du code, une comparaison ou conférence des articles, et une table alphabétique et analytique des matières. Montréal: C. Théoret, I 897. xxxi, 394, lxxxvii p.

II Loc.: ICMH, no 298I4; QMBN

70. DORAIS, O.P. (Oscar-Pierre); DORAIS, A.P. (Albert-Paul).

[Code de procédure civile (1903)]

Code de procédure civile de la province de Québec revu, augmenté et mis au courant de la législation comprenant les observations spéciales des commissaires chargés de la révision et modification du Code de procédure civile du Bas-Canada, une table de concor- 
dance des articles entre l'ancien code et le code actuel, les lois et autorités qui ont été la base du code actuel, une comparaison ou conférence des articles du code entre eux et avec ceux du code civil, et des renvois aux statuts et aux règles de pratique, avec un appendice comprenant les matières en rapport avec le code de procédure, la loi de conciliation, et les règles de pratique, les tarifs d'honoraires des officiers des différents tribunaux civils de la Province de Québec, le tarif des régistrateurs, les tarifs d'honoraires des avocats et des notaires et une table alphabétique et analytique des matières. 2e éd. Montréal: C. Théoret, I903. xi, 66I, cxxv p.

II Loc.: QMBN; QMM

7I. DORAIS, O.P. (Oscar-Pierre); DORAIs, A.P. (Albert-Paul).

Formulaire de procédure de la province de Québec comprenant un grand nombre de formules relatives au Code de procédure civile, aux règles de pratique, et au Code civil, contenant aussi des formules relatives à la Loi du barreau et aux Statuts refondus de Québec. Montréal: C. Théoret, I901. xii, 583 p.: formules.

II LOc.: QMBN; QQLA

72. GERMANO, $\mathrm{J}$.

Nouveau formulaire des actes des notaires de la province de Québec. Montréal: C. Théoret, I903. viii, 478 p.: formules.

II LOC.: QMBN; QQLA

73. HOLT, Chas. M. (Charles Macpherson)

A treatise on the insurance law of Canada embracing fire, life, accident, guarantee, mutual benefit, etc. with an analysis of the jurisprudence and of the statute law of the Dominion. Montréal: C. Théoret, r 898. $x x x i, 878 \mathrm{p}$.

II LOC.: ICMH, no IO62I; OONL; QQLA

74. KaVANAGH, Henry John.

The legal incapacity of wives separate as to property in the light of the dicision (sic) of Privy Council in the case of the Trust and Loan Co., of Canada and Dame H. de Kerouack: a paper. Montréal: C. Théoret, 1903. ro p.

II Loc.: QMBN

75. LAFLEUR, E. (Eugène).

The conflict of laws in the province of Quebec. Montreal: C. Théoret, r898. xvi, $267 \mathrm{p}$.

II Loc.: ICMH, no I0704; QMBN; QQLA

76. LAGRANGE, E. (Eugène).

Manuel de droit romain ou Explication des institutes de Justinien par demandes et par réponses précédé d'une introduction historique à l'étude du droit romain et d'une bibliothèque choisie de ce droit. I 3 e éd. rev. Montréal: C. Théoret, I897. 616 p.

II LOC.: ICMH, no 38I23; OONL; QQLA 
77. LANGELIER, François.

De la preuve en matière civile et commerciale, avec un index alphabétique et analytique de tout l'ouvrage. Montréal: C. Théoret, I 895. xxiii, $437 \mathrm{p}$.

II Loc.: QQLA

78. LEMIEUX, Rodolphe.

De la contrainte par corps: thèse pour le doctorat présentée et soutenue le rer mai I 896. Montréal: C. Théoret, I896. iv, I99 p.

II LOC.: ICMH, no 8605; OONL; QMBN; QQLA

79. LEMIEUX, Rodolphe.

Les origines du droit franco-canadien, comprenant: - I L'Histoire du droit français depuis l'époque gallo-romaine jusqu'à l'ère révolutionnaire inclusivement; - II L'Histoire du droit canadien depuis la découverte du Canada jusqu'à la cession (domination française), et depuis le Traité de Paris (I763) jusqu'à la Confédération inclusivement (domination anglaise). Montréal: $\mathrm{C}$. Théoret, r900. xxix, $483 \mathrm{p}$.

II Loc.: ICMH, no 8604; OONL; QMBN; QQLA

Cet ouvrage fut réimprimé en 1901 .

80. LEMIEUX, Rodolphe.

Wilfrid Laurier: conférence devant le Club national de Montréal.

Montréal: C. Théoret, I897. 58 p.

II Loc.: ICMH, no 8606; QMBN

8I. Martineau, Paul G.; Delfausse, Romuald.

[Code de procédure civile (I 899). Français et anglais]

Code de procédure civile de la province de Québec annoté avec les textes anglais et français en regard, suivi d'un appendice contenant les formules de ce Code, les matières en rapport avec le Code de procédure, la Loi de conciliation et les règles de pratique des différents tribunaux. Montréal: C. Théoret, I 899. 1xv, 91 2, xxxvii p.: formules.

II LOC.: QMBN; QQLA

82. Martineau, Paul G.; Delfausse, Romuald.

[Code de procédure civile (1903). Supplément]

Supplément au Code de procédure civile annoté de la province de Québec, contenant la jurisprudence et les amendements depuis la publication du Code annoté de I899. Montréal: C. Théoret, I903. xxiii, $220 \mathrm{p}$.

II Loc.: QMBN; QQLA

83. MASSICOTTE, E.Z. (Édouard-Zotique).

Le droit civil canadien résumé en tableaux synoptiques d'après la méthode de A. Wilhelm. Montréal: C. Théoret, I896. I28 p.

II Loc.: ICMH, no 9966; OONL; QMBN; QQLA

84. Olivier, Arthur.

Manuel de la Cour des commissaires de la province de Québec pour la décision sommaire des petites causes avec textes anglais et 
français.Montréal: C. Théoret, 1902. xvi, I6I p.

II Loc.: QQLA

85. PATterson, William.

Handbook of commercial law. Montréal: C. Théoret, I905. viii, 202 p.: formules.

II LOc.: QMBN; QQLA

86. PERRON, J.L. (Joseph-Lucien); MITCHELL, Victor E. (Victor-Evelyn).

Manuel des faillites comprenant le texte français et anglais des articles du Code de procédure civile sur la cession de biens, le capias ad respondendum, la saisie-arrêt avant jugement, la saisie revendication, la saisie conservatoire, et la liquidation des sociétés, ainsi qu'un exposé des changements apportés à l'ancienne loi par la revision du Code de procédure civile. Montréal: C. Théoret, I898. xii, I44 p.: formules.

II LOc.: OONL; QMBN; QQLA

87. ROY, J. Edmond (Joseph-Edmond).

L'ancien barreau au Canada; conférence donnée devant le Barreau de Québec dans la salle de la Cour d'assises au mois de février I897. Montréal: C. Théoret, I897. 9I p.

II Loc.: ICMH, no I 272I; QMBN; QQLA

88. Refonte de la Loi Augé sur les privilèges sur les immeubles, quant à ce qui concerne spécialement l'architecte, le constructeur, l'ouvrier et le journalier. [Compilation] par Lavallée, Lavallée \& Lavallée, avocats au Barreau de Montréal. Montréal: C. Théoret, I897. I 5 p. II Loc.: QMBN

89. Rules of practice of the Superior Court, the Circuit Court and the Court of Review. Containing an alphabetic index by Dorais \& Dorais. Montréal: C. Théoret, I898. I I4 p.

II LOC.: OONL

90. SAINT-PIERRE, H.C. (Henri-Césaire).

Affaire de W.A. Grenier propriétaire du journal 'La libre parole', accusé de libelle par l'Honorable J. Israel Tarte, ministre des Travaux publics. Plaidoyer de Mtre H.C. St-Pierre, C.R., pour la poursuite suivi du résumé des débats par l'Hon. juge Wurtele. Montréal: C. Théoret, [1897?]. x, I45 p.

II LOC.: ICMH, no I 3I 25; OONL; QMBN

91. SaUvalle, Marc.

La loi de conciliation: guide du conciliateur, explication détaillée avec formules usuelles de la loi 62 Victoria, chap. 54, entrée en vigueur le Io mai I 899. Montréal: C. Théoret, I899. xvi, I04 p.

II Loc.: ICMH, no I 3097; QMBN; QQLA

92. SHARP, William Prescott.

[Code civil (I 895). Supplément anglais]

Consolidated supplement no I to Sharp's Civil Code, containing all statutory enactments and a digest of all reported cases affecting the Civil Code of Lower Canada from the Ist October I888 to I4th 
October I895. Montréal: C. Théoret, I 896. vii, 704 p.

II Loc.: OONL; QMBN

Cet ouvrage fut réimprimé en 1898 .

93. TASCHEREAU, Robert.

Théorie du cas fortuit et de la force majeure dans les obligations: thèse pour le doctorat présentée et soutenue le s octobre I90I. Montréal: C. Théoret, r9or. vii, I64 p.

II Loc.: QMUM

94. WALton, Frederick Parker.

The new laws of employers' liability in England and France and their bearing on the law of the province of Quebec with the text of the two acts. Montréal: C. Théoret, I900. vi, $67 \mathrm{p}$.

II Loc.: ICMH, no 36739; OONL; QQLA

95. WEIR, Robert Stanley.

[Code civil (1 898). Anglais]

The Civil Code of Lower Canada and the Bills of Exchange Act, I890, with all statutory amendments verified, collated and indexed. Montréal: C. Théoret, I898. ix, 462 p.: formules.

II Loc.: ICMH, no 9287; OONL; QMBN; QQLA

96. WEIR, Robert Stanley.

[Code de procédure civile (I 900). Anglais]

The Code of Civil Procedure of the province of Quebec with a concordance of its articles, useful references to the Civil Code, and the rules of practice now in force-The whole carefully verified and edited. Montréal: C. Théoret, I900. 30, 303 p.: formules.

II Loc.: ICMH, no 2574I; OONL; QMBN; QQLA

97. WEIR, Robert Stanley.

[Code municipal (1903). Anglais]

The Municipal Code of the province of Quebec (annotated) containing all the judgments of the courts, an historical sketch of our municipal institutions, also an appendix giving the Quebec License Act, the Quebec Election Act and the law relating to jurors and juries. Montréal: C. Théoret, I903. xlvi, $453 \mathrm{p}$.

II LOc.: QMBN; QQLA

98. WEIR, Robert Stanley.

[Loi de l'Instruction publique (1 899). Anglais]

The Education Act of the Province of Quebec [62 Victoria, cap. 28], with all the relevant decisions of the courts, and the regulations of the Protestant and Roman Catholic Committees of the Council of Public Instruction compiled, collated and indexed. Montréal: C. Théoret, I 899. xxx, 192, 52 p.: formules.

II Loc.: ICMH, no 42627; QQLA

99. White, W.J.; EWING, J.A.

A treatise on Canadian company law containing a commentary on the Companies Act of the Dominion, with incidental reference to the law of the various provinces with full notes of the jurispru- 
dences and appendices of the statutes and useful forms. Montréal:

C. Théoret, I901. xxiii, 708 p., formules.

II LOC.: QMBN; QQLA

Ioo. WILHeLM, A. (Albert).

Le droit romain résumé en tableaux synoptiques. 9e éd. rev. et augm. Montréal: C. Théoret, I 897.87, 98 p.

II LOc.: ICMH, no 35805 ; QQLA

IOI. WÜRTELE, Jonathan Saxton Campbell.

Adresse de l'hon. juge Würtele aux petits jurés, le 2 octobre I897, et allocution au défendeur lors de la sentence, le I4 octobre I 897, dans le procès pour libelle de la Reine vs. W.A. Grenier. Montréal: C. Théoret, [1897?]. $38 \mathrm{p}$.

II LOc.: ICMH, no 26285; OONL

\section{Ouvrages édités par W.J. Wilson (1897-1901)}

I02. BAUdouin, Ph. (Philibert).

Index to incorporated bodies and to private and local law under Dominion, and Manitoba, Ontario and Quebec statutes, proclamations and letters patent shewing in one alphabetical order nearly I sooo names from I7 Geo. III (I777) to Ist January I 896. Montréal: W.J. Wilson, I897. xiii, 709 p.

II Loc.: QMBN

IO3. LANCTOT, Charles.

[Code criminel (I90I). Anglais]

The Criminal Code of Canada as in force on January Ist, I90I with the reported cases and appendices, containing: the Canada Evidence Act and the Extradition Act and decisions, a list of the extradition treaties, the statutes concerning fugitive offenders, Habeas Corpus, identification of criminals, conditional liberation of prisoners, militia in aid of civil power, coroners, etc. Montréal: W.J. Wilson, I 901 . lxiv, 823 p.: formules.

II Loc.: QMBN; QQLA

E. Ouvrages édités par Wilson et Lafleur ass. (1905-1909)

I04. BEAUCHAMP, J.J. (Jean-Joseph).

The jurisprudence of the Privy Council containing a digest of all the decisions of the Privy Council since the publication of the first volume in I89I; the amendments to the constitution of the Judicial 
Committee, and the new rules of practice and also two appendices. Montréal: Wilson et Lafleur, 1909. xiv, 452 p.

II Loc.: QMBN; QQLA

105. BEAUChamp, J.J. (Jean-Joseph).

Répertoire de la Revue légale, N.S., et de la Revue de jurisprudence sous forme alphabétique et chronologique contenant un résumé des décisions judiciaires canadiennes et étrangères avec les noms de la cour, des juges et des parties, la date du jugement et les autorités citées, ainsi que les écrits publiés dans les 24 derniers volumes de ces revues, suivis d'une table des causes. Montréal: Wilson et Lafleur, I 908. Xx, 2557 p.

II Loc.: QQLA

I06. BÉDARD, J.É. (Joseph-Édouard).

[Code municipal (1905). Français et anglais]

Code municipal de la province de Québec annoté, mis au courant de la législation et de la jurisprudence, et suivi des dispositions statutaires concernant les officiers municipaux quant aux élections parlementaires, licences, jurés, $₫ c .2 \mathrm{e}$ éd. Montréal: Wilson et Lafleur, I905. xxix, 715 p.: formules.

II Loc.: QMBN

107. BERNARD, Mathieu A. (Mathieu-Adolphe).

Manuel de droit commercial théorique et pratique de la Province de Québec. ze éd. par J.-F. Saint-Cyr. Montréal: Wilson et Lafleur, I906. I $32 \mathrm{p}$.

II Loc.: QMBN; QQLA

IO8. CAMERON, Edward Robert.

The law of fire insurance in Canada, with a complete analysis of the jurisprudence and of the statute law of the Dominion. Montréal: Wilson et Lafleur, I909. xxxiv, 630 p.

II Loc.: QMBN; QQLA

IO9. CRANKSHAW, James.

[Code criminel (1907). Supplément. Anglais]

Supplement to the Criminal Code and the Canada Evidence Act.

Montréal: Wilson et Lafleur, I907. xxii, 44I p.: formules.

II Loc.: QMBN

IIO. LANGELIER, François.

Cours de droit civil de la province de Québec. Montréal: Wilson et Lafleur, I905-I9II. $6 \mathrm{v}$.

II Loc.: QMBN; QQLA

III. MCMAHON, Edmond.

A practical guide to the coroner and his duties at inquests without and with a jury, in Quebec, and other provinces of Canada. Montréal: Wilson et Lafleur, I907. xxii, 370 p.

II Loc.: QMBN

II 2. SAINT-CYR, J.F. (Joseph-Fortunat).

Index-digest to the Montreal law reports, arranged in the alphabet- 
ical and chronological order containing in each decision the name of the courts, the judges and the interested parties, the date of judgment and the authorities cited; also a table of cases reported. Montréal: Wilson et Lafleur, I905. 538 p.

II Loc.: QQLA

II 3. SIROIS, Joseph.

De la forme des testaments: thèse pour le doctorat. Montréal:

Wilson et Lafleur, I907. 398 p.

II Loc.: QMBN; QQLA

II4. WALton, Frederick Parker.

The scope and interpretation of the Civil Code of Lower Canada. Montréal: Wilson et Lafleur, 1907. xix, 159 p.

II Loc.: QQLA

\section{F. Ouvrages édités par Wilson et Lafleur ltée (1910-1920)}

i 5. ARchambault, A.S.

[Code de procédure civile (I920)]

Code de procédure civile de la province de Québec revu et mis au courant de la législation jusqu'au i 4 février I 920 comprenant la loi relative à l'organisation des tribunaux dans la province (Io Geo. V, c. 79), les règles de pratique en vigueur dans les cours provinciales et une table alphabétique et analytique des matières. $4 \mathrm{e}$ éd. de l'édition du Code de procédure civile de Dorais et Dorais. Montréal: Wilson et Lafleur, I920. xxxi, 566 p.: formules.

II Loc.: QMBN; QMM

II6. BEAUCHAMP, J.J. (Jean-Joseph).

Répertoire général de jurisprudence canadienne contenant un résumé, sous forme alphabétique et chronologique, de toutes les décisions judiciaires rapportées du Conseil privé, de la Cour suprême, de la Cour de l'échiquier, des cours d'amirauté, de la Commission des chemins de fer et des tribunaux de la province de Québec et de toute la puissance du Canada dans tout ce qui tombe sous la juridiction du parlement fédéral, depuis 1770 jusqu'à mai I913, ainsi qu'une référence aux matières qui se trouvent dans les statuts fédéraux et provinciaux et le texte de ces lois se rapportant au droit civil, avec divers appendices. Montréal: Wilson et Lafleur, I9I4. $4 \mathrm{v}$.

II Loc.: QQLA

II7. BOUCHETTE, Errol.

L'indépendance économique du Canada français. ze éd. Montréal: Wilson et Lafleur, I9I3. 293 p.

II Loc.: QMBN 
II8. BUtLeR, Wm. H.

[Code civil (I9 Io). Anglais]

The Civil Code of Lower Canada and the Bills of Exchange Act, I906, with all statutory amendments verified, collated and indexed. Montréal: Wilson et Lafleur, r910. vi, 592 p.

II Loc.: QQLA

II9. CAMERON, Edward Robert.

The law of life, accident 4 guarantee insurance in Canada, with a complete analysis of the jurisprudence and statute law of the Dominion and of the different provinces. Montréal: Wilson et Lafleur, 1910. xxxi, ro52 p.: formules.

II Loc.: QMBN; QQLA

I2O. DEGUIRE, A.S.

[Code civil (I919)]

Code civil de la province de Québec. 4e éd. de l'édition du Code civil de Dorais et Dorais. Montréal: Wilson et Lafleur, 1919. xxix, 676 p.: formules.

II Loc.: QMBN; QMM

I2I. FORAN, T.P. (Thomas Patrick).

[Loi des accidents du travail (I 9Io). Français et anglais]

The Workmen's Compensation Act of Quebec. Montréal: Wilson et Lafleur, I910. xi, $230 \mathrm{p}$.

II LOC.: QMBN; QQLA

I22. GÉRIN-LAJOIE, Henti.

[Code de procédure civile (I920). Français et anglais]

Code de procédure civile de la province de Québec annoté avec les textes français et anglais contenant la jurisprudence sous chaque article et accompagné des règles de pratique annotées des tribunaux de la province de Québec, du rapport des commissaires chargés de la révision du code et de la 'Loi relative à l'organisation et à la compétence des tribunaux de juridiction civile' (ro Geo. V, c. 79). Montréal: Wilson et Lafleur, I920. xiv, 2018 p.

II LOC.: QMBN; QQLA

123. JOHNSON, Walter $S$.

[Code civil (I9 I 8). Anglais]

Civil Code of Lower Canada, with the amendments effected by imperial, federal and provincial legislation, up to and including the second session of the fourteenth legislature of the province of Quebec, 8 George V, I9I 8, also the Federal Bills of Exchange Act as amended to date. Montréal: Wilson et Lafleur, I9I8. xxviii, 939 p.: formules.

II Loc.: QMBN; QMM

I24. LORD, Fortunat.

[Code scolaire (1912)]

Code scolaire de la province de Québec annoté: cet ouvrage renferme la loi scolaire revisée, les origines de chacun de ses 
articles, des références aux codes de cette province, les règlements du Comité catholique, l'ancienne jurisprudence et la nouvelle. Montréal: Wilson et Lafleur, I9 I 2. viii, 580 p.: formules.

II Loc.: QMBN; QQLA

I25. LORD, Fortunat.

[Code scolaire (19r9)]

Code scolaire de la province de Québec annoté: cet ouvrage renferme la loi scolaire revisée, les origines de chacun de ses articles, des références aux codes de cette province, les règlements du Comité catholique, l'ancienne jurisprudence et la nouvelle. Montréal: Wilson et Lafleur, I9I2 [I9I9]. viii, I3, 580 p.: formules.

II Loc.: QMBN

I26. LORD, Fortunat.

[Loi des liquidations (r9r3)]

The Dominion Winding-up Act. Montréal: Wilson et Lafleur, I 9 I 3. xxviii, $301 \mathrm{p}$.

II Loc.: QMBN

I27. LORTIE, ?

[Lois municipales (I 919)]

Emprunts municipaux: lois qui les régissent, formulaire des procédures requises pour l'approbation des règlements d'emprunts municipaux. Montréal: Wilson et Lafleur, 1919. I67 p.: formules.

II Loc.: QMBN

I28. MARSAN, G.A. (Georges-Avila).

Code des médecins et chirurgiens, droits et obligations professionnels des médecins, dentistes, pharmaciens, vétérinaires, opticiens, étudiants et loi des coroners. Montréal: Wilson et Lafleur, I920. xiv, 678 p.: formules.

II Loc.: QMBN; QQLA

I29. MERRILL, Walter A.

[Loi des accidents du travail (I9I6). Français et anglais]

The Workmen's Compensation Act of Quebec and reported decisions (cases reported to Ist, January 1916). Montréal: Wilson et Lafleur, I9I6. X, $69 \mathrm{p}$.

II Loc.: QMBN

I30. SAINT-CYR, J.F. (Joseph-Fortunat).

Loi des licences de Québec annotée (Quebec Liquor License Law) comprenant toute la législation concernant les liqueurs envirantes, les références des différents articles entre eux, les jugements rendus sur cette matière dans la Puissance du Canada et les concordances avec les lois des licences des autres provinces et avec l'Acte de tempérance du Canada. Montréal: Wilson et Lafleur, I9I I. viii, $383 \mathrm{p}$. II Loc.: QMBN

I 3 I. SAINT-CYR, J.F. (Joseph-Fortunat).

La loi pour tous. Montréal: Wilson et Lafleur, I9I2. viii, 23 I p.: formules. 
II LOc.: QMBN; QQLA

I32. SURVEYER, E.F. (Édouard Fabre).

[Code de procédure civile (I915)]

Code de procédure civile de la province de Québec, revu, augmenté et mis au courant de la législation, les règles de pratique en vigueur dans les cours provinciales et une table alphabétique et analytique des matières. 3e éd. de l'édition du Code de procédure civile de Dorais et Dorais. Montréal: Wilson et Lafleur, I9I 5 . xxiii, 520 p.: formules.

II Loc.: QMBN; QMM

I33. SURVEYER, E.F. (Édouard Fabre).

[Code de procédure civile (I9I7)]

Code de procédure civile de la province de Québec, revu, augmenté et mis au courant de la législation, les règles de pratique en vigueur dans les cours provinciales et une table alphabétique et analytique des matières. Montréal: Wilson et Lafleur, I9I5 [r9I7]. 520 p.: formules:

II Loc.: QMBN

\section{Catalogue de libraires ${ }^{\dagger}$}

134. Catalogue of Law Books, Canadian, French, English and American: Imported and for Sale by $A$. Périard. Catalogue de livres de droit canadien, français, anglais et américain. Montréal: A. Périard, I883. $36 \mathrm{p}$.

II Loc.: Lamonde, p. 152 , no 25

135. Periard's New Catalogue of Law Books Relating Chiefly to the Jurisprudence of the Province of Quebec/Nouveau catalogue de la librairie de droit et de jurisprudence de la province de Québec. Montréal: A. Périard, I 885.48 p.

II Loc.: Lamonde, p. I 53, no 27. Le seul exemplaire signalé semble disparu.

136. Nouveau catalogue de livres de droit et de jurisprudence de la librairie A. Périard, français-anglais-américain et canadien. Montréal: A. Périard, I890. 47 p.

II Loc.: OTYL

137. Nouveau catalogue de livres de droit et de jurisprudence de la librairie A. Périard: français-anglais-américain et canadien. Montréal: A. Périard, r891. 62 p.

II Loc.: ICMH, no 45903

† Cette liste a été établie à partir de l'ouvrage de Y. Lamonde; on se référera donc à son catalogue pour les localisations (La librairie et l'édition à Montréal, I776-199I) (Montréal: Bibliothèque nationale du Québec, I99I). Les ajouts sont de l'auteur. 
138. Catalogue de livres de droit, Whiteford è Théoret, libraires - éditeurs - imprimeurs e relieurs/Catalogue of Law Books, Whiteford $\Theta$ Théoret, Publishers - Booksellers - Importers थ Binders. Montréal: Whiteford-Théoret, [c. I 894]. 132 p.

II Loc.: Lamonde, p. I 56, no 39

139. A Catalogue of Canadian Law Books Arranged Under the Author's Names, Published by C. Théoret, Law Bookseller, Importer and Binder also an List of other Canadian Law Publications and Recent English and American Law Works. Montréal: C. Théoret, 1899. $67 \mathrm{p}$.

TI Loc.: Lamonde, p. I 57, no 44

140. Catalogue de livres de droit et de jurisprudence de la Province de Québec (ci-devant Bas-Canada) avec table alphabétique des matières. Montréal: C. Théoret, I900. 48-viii p.

II Loc.: ICMH, no I 494I; Lamonde, p. I 58, no 47

I4I. Catalogue of Law Books Published in Canada, Great Britain, France and United States ... Montréal: C. Théoret, 1902, $84 \mathrm{p}$.

II Loc.: Lamonde, p. I 59, no 49

142. Catalogue of Law Books Published in Canada, Great Britain, France and United States, Classified Alphabetically Under the Named of Authors, Followed with an Alphabetical Index of Subjects. Montréal: C. Théoret, I905. I I I p.

II Loc.: Lamonde, p. I59, no 50 
